\title{
Prediction and Validation of Gene-Disease Associations Using Methods Inspired by Social Network Analyses
}

\author{
U. Martin Singh-Blom ${ }^{1,29}$, Nagarajan Natarajan ${ }^{39}$, Ambuj Tewari ${ }^{4}$, John O. Woods ${ }^{1}$, Inderjit S. Dhillon ${ }^{3 *}$, \\ Edward M. Marcotte ${ }^{1,5 *}$
}

1 Center for Systems and Synthetic Biology, Institute for Cellular and Molecular Biology, University of Texas, Austin, Texas, United States of America, 2 Department of Medicine, Karolinska Institutet, Solna, Stockholm, Sweden, 3 Department of Computer Science. University of Texas, Austin, Texas, United States of America, 4 Department of Statistics. University of Michigan, Ann Arbor, Michigan, United States of America, $\mathbf{5}$ Department of Chemistry and Biochemistry. University of Texas, Austin, Texas, United States of America

\begin{abstract}
Correctly identifying associations of genes with diseases has long been a goal in biology. With the emergence of large-scale gene-phenotype association datasets in biology, we can leverage statistical and machine learning methods to help us achieve this goal. In this paper, we present two methods for predicting gene-disease associations based on functional gene associations and gene-phenotype associations in model organisms. The first method, the Katz measure, is motivated from its success in social network link prediction, and is very closely related to some of the recent methods proposed for genedisease association inference. The second method, called CATAPULT (Combining dATa Across species using Positive-Unlabeled Learning Techniques), is a supervised machine learning method that uses a biased support vector machine where the features are derived from walks in a heterogeneous gene-trait network. We study the performance of the proposed methods and related state-of-the-art methods using two different evaluation strategies, on two distinct data sets, namely OMIM phenotypes and drug-target interactions. Finally, by measuring the performance of the methods using two different evaluation strategies, we show that even though both methods perform very well, the Katz measure is better at identifying associations between traits and poorly studied genes, whereas CATAPULT is better suited to correctly identifying gene-trait associations overall. The authors want to thank Jon Laurent and Kris McGary for some of the data used, and Li and Patra for making their code available. Most of Ambuj Tewari's contribution to this work happened while he was a postdoctoral fellow at the University of Texas at Austin.
\end{abstract}

Citation: Singh-Blom UM, Natarajan N, Tewari A, Woods JO, Dhillon IS, et al. (2013) Prediction and Validation of Gene-Disease Associations Using Methods Inspired by Social Network Analyses. PLoS ONE 8(5): e58977. doi:10.1371/journal.pone.0058977

Editor: Patrick Aloy, Institute for Research in Biomedicine, Spain

Received September 24, 2012; Accepted February 12, 2013; Published May 1, 2013

Copyright: (C) 2013 Singh-Blom et al. This is an open-access article distributed under the terms of the Creative Commons Attribution License, which permits unrestricted use, distribution, and reproduction in any medium, provided the original author and source are credited.

Funding: This work was supported by grants from the U.S. Army Research (58343-MA) to EMM and ISD, from the Cancer Prevention \& Research Institute of Texas (CPRIT), U.S. National Science Foundation, United States National Institutes of Health, Welch Foundation (F1515), and the Packard Foundation to EMM, and from DOD Army (W911NF-10-1-0529), U.S. National Science Foundation (CCF-0916309) and the Moncrief Grand Challenge Award to ISD. The funders had no role in study design, data collection and analysis, decision to publish, or preparation of the manuscript.

Competing Interests: The authors have declared that no competing interests exist.

*E-mail: marcotte@mail.utexas.edu (EMM); inderjit@cs.utexas.edu (ISD)

9 These authors contributed equally to this work.

\section{Introduction}

Correctly predicting new gene-disease associations has long been an important goal in computational biology. One very successful strategy has been the so-called guilt-by-association (GBA) approach, in which new candidate genes are found through their association with genes already known to be involved in the condition studied. This association can in practice be derived from many different types of data. Goh et al.[1] construct a network where genes are connected if they are associated with the same disease, whereas Tian et al.[2] combine protein interactions, genetic interactions, and gene expression correlation, and Ulitsky and Shamir[3] combine interactions from published networks and yeast two-hybrid experiments.

One of the most commonly used kinds of association is derived from direct protein-protein interactions, such as the ones curated by the Human Reference Protein Database (HPRD) [4]. The last few years have seen a number of methods, such as CIPHER [5], GeneWalker [6], Prince [7] and RWRH [8], that have extended the association from just direct protein interactions to more distant connections in various ways. One kind of network that has proven to be particularly useful for predicting biological function is the functional interaction network, where a pair of genes is connected based on the integrated evidence from a wide array of information sources, as seen by Lee at al.[9]. These have been used to associate genes with phenotypes in model organisms $[10,11]$ and in humans $[12,13]$. A recently published network, HumanNet, has been used to refine predictions from genome-wide association studies [14]. Since functional gene interaction networks aggregate many different types of information, they can achieve much greater coverage than pure protein-protein interaction networks.

Alternatively, we can think of the gene-disease association problem as a supervised learning problem, where each gene-disease pair is represented by a number of derived features (explicitly or implicitly using a kernel function) and then a classifier is learned to distinguish "positive" (or known) associations from "negative" ones, using previously studied gene-disease associations, and unknown gene-disease pairs as training data. Such an approach is taken by 
the recent ProDiGe method [15], which integrates a wide variety of heterogeneous data sets and uses support vector machines (SVMs) to identify potential gene-disease associations.

In the past decades, the growth of gene-phenotype associations in model species has been explosive, which suggests an alternative way to find candidate genes for human diseases. McGary et al.[16] used this treasure trove of information to find surprising connections between model species phenotypes and human diseases by looking for pairs of human diseases and model phenotypes that share a higher than expected number of orthologous genes. In this way, a number of new, and often surprising, model systems were found for human diseases. For instance, the human neural crest related developmental disorder Waardenburg syndrome shares gene modules with gravitropism (the ability to detect up and down) in plants, and mammalian angiogenesis has been found to involve the same pathways as lovastatin sensitivity in yeast. This model species information represents yet another form of functional connection that can be used for gene-phenotype association.

In this paper, we first propose two distinct but related GBA methods. One is based on the Katz method [17] that has been successfully applied for link prediction in social networks. The method is based on integrating functional gene interaction networks with model species phenotype data and computing a measure of similarity based on walks of different lengths between gene and phenotype node pairs. The second method, which we call Catapult (Combining dATa Across species using PositiveUnlabeled Learning Techniques) is a supervised learning method, wherein we represent gene-phenotype pairs in a feature space derived from hybrid walks through the heterogeneous network used by Katz. The supervised learning method falls under a class of learning methods called Positive-Unlabeled learning methods (ProDiGe [15] also belongs in this class) since the learning task has only positive and unlabeled examples (and no negative examples). The method naturally generalizes the computation of Katz on a heterogeneous network by learning appropriate feature weights.

To determine if a computational method truly associates genes with diseases, biological validation of the predicted associations often by knockout studies in model systems, or through sequencing of patients - is needed. Since these can be expensive and hard to do in a high throughput way, it is common to measure the performance of GBA methods through cross-validation. Recent work has shown that a large fraction of the performance of GBA methods can be attributed to the multifunctionality of genes [18]. A priori, it is not clear exactly how the construction of the training and the test data sets affects the measured performance of a method. We show that Katz and Catapult outperform the stateof-the-art, as measured by standard cross-validation. Furthermore, we show that standard cross-validation is not always an appropriate yardstick for comparing the performance of methods, and that when an alternative method for cross-validation is used - measuring how well the methods do in predicting genes that have no previous disease (or drug) associations, simpler walk-based methods often achieve better performance than supervised learning counterparts. We also observe that the qualitative performance of the methods correlates better with the latter evaluation strategy. We evaluate the two proposed methods, and compare to state-of-the-art network-based gene-disease prediction approaches on two completely distinct sources of data, namely OMIM phenotypes and gene-drug interactions.

\section{Results and Discussion}

Conceptually, gene-disease association data can be thought of as a bipartite graph, where each gene and each disease is a node, and there is an edge between a gene node and a disease node if there is a known association between the gene and the disease. Similarly, we can form bipartite graphs from gene-phenotype association data of different species. By connecting a phenotype with a human gene if any ortholog of the human gene is associated with the phenotype, we obtain a bipartite network between human genes and phenotypes of different species. We can also obtain a phenotype-phenotype network for a given species, where a (weighted) edge $(i, j)$ indicates that phenotype $i$ is "similar" to phenotype $j$. Adding a gene-gene interaction network completes a heterogeneous network of human genes and phenotypes in a wide variety of species. It is straightforward to define analogous heterogeneous network for gene-drug interactions, by replacing gene-disease associations data of humans with gene-drug associations. More limited heterogeneous networks have been considered previously in the context of gene-disease predictions, like the network of protein-protein interactions and human diseases [8], and in the context of gene-drug predictions [19]. Through a holistic view of the networks, otherwise unobserved ways of interactions between genes are revealed (via shared phenotypes), and independent information hidden in the model organism data can be leveraged for discovering novel associations between genes and human diseases or drugs. By integrating functional information from orthologs in multiple species, we also implicitly encode the functional relationships between homologous genes in the humans, which also contributes to our predictive performance. A visualization of the heterogeneous network consisting of gene-gene network and gene-phenotype networks of a few model species is presented in Figure 1.

In this setting, it is natural to view the problem of predicting gene-phenotype associations as a problem of finding similarities between nodes in a heterogeneous graph. Posing the problem in this way comes with the significant advantage that we can leverage a large body of work in machine learning and network analysis that deals with the problem of finding similar nodes in a graph [20,21]. In particular, we adapt the Katz method [20] to the heterogeneous setting. As an extension of this work, we also introduce a supervised learning framework, Catapult. Catapult learns the importance of features associated with node pairs, where the features are derived from walk-based similarity measures between nodes.

\section{Katz on the heterogeneous network}

The Katz measure is a graph-based method for finding nodes similar to a given node in a network[17]. It has been shown to be successful for predicting friends in social networks [20]. In this paper, we show the effectiveness of the method for the task of recommending genes for a given phenotype or drug. Suppose we are given an undirected, unweighted graph with a (symmetric) adjacency matrix $A$, where $A_{i j}=1$ if node $i$ and node $j$ are connected, and $A_{i j}=0$ otherwise. One way to find the similarity between two (not necessarily connected) nodes $i$ and $j$ is to count the number of walks of different lengths that connect $i$ and $j$. This has a natural connection to matrix powers since $\left(A^{l}\right)_{i j}$ is exactly the number of walks of length $l$ that connect $i$ to $j$. Hence $\left(A^{l}\right)_{i j}$ gives a measure of similarity between the nodes $i$ and $j$. We want to obtain a single similarity measure that summarizes the similarities suggested by different walk lengths. For example, we could choose any sequence $\beta_{l}$ of non-negative coefficients and define the similarity 


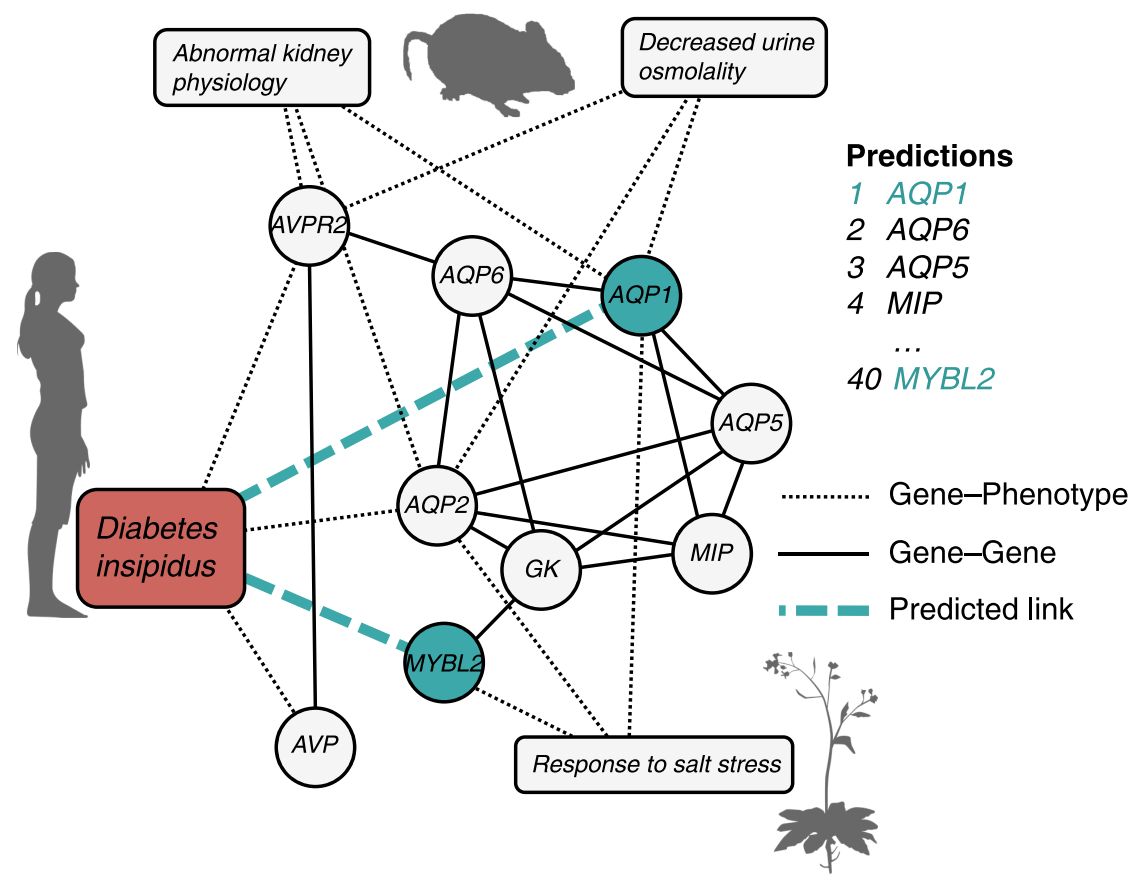

Figure 1. The combined network in the neighborhood of a human disease. The local network around the human disease diabetes insipidus and two genes highly ranked by CATAPULT, AQP1 (top ranked candidate) and MYBL2 (ranked as number 40). AQP1 is ranked higher than MYBL2 because there are more paths from diabetes insipidus to $A Q P 1$ than to $M Y B L 2$, both through model organism phenotypes and through the gene--gene network. Only genes and phenotypes that are associated to both diabetes insipidus and the predicted genes AQP1 and MYBL2 are shown.

doi:10.1371/journal.pone.0058977.g001

$$
S_{i j}=\sum_{l=1}^{k} \beta_{l}\left(A^{l}\right)_{i j}
$$

where $\beta$ is a constant that dampens contributions from longer walks. In matrix notation, the similarity matrix $S$ (that captures similarities between all pairs $i$ and $j$ ) may be written as:

$$
S=\sum_{l=1}^{k} \beta_{l} A^{l} .
$$

As observed by a recent survey article [21], we can regard $S$ as a matrix function $F(A)$ where $F$ is defined through the series expansion in (1). Note that we may allow $k \rightarrow \infty$, as long as $\beta_{l} \rightarrow 0$ as $l \rightarrow \infty$. Specific choices for $\beta_{l}$ yield a variety of concrete similarity measures. A choice of $\beta_{l}=\beta^{l}$ leads to the well-known Katz measure [17]:

$$
S^{k a t z}=\sum_{l \geq 1} \beta^{l} A^{l}=(I-\beta A)^{-1}-I,
$$

where $\beta$ is chosen such that $\beta<\frac{1}{\|A\|_{2}}$. In the case where the connections in the graph are weighted such that $A_{i j}$ is the strength of the connection between nodes $i$ and $j$, we can generalize the idea of walks using this matrix framework, by simply using the weighted adjacency matrix instead of the binary matrix. Different ways of constructing the matrix $A$ together with the appropriate normalizations of the matrix lead to methods of the type used by PRINCE [7], RWRH [8], GeneMANIA [11], and by the famous PageRank algorithm used for web page ranking [22]. However, we do not necessarily have to consider sum over infinitely many path lengths. Paths of shorter lengths often convey more information about similarity between a given pair of nodes, and contributions from longer paths become insignificant. This suggests that we can consider a finite sum over path lengths, and typically small values of $k(k=3$ or $k=4)$ are known to yield competitive performance in the task of recommending similar nodes [23].

Let $G$ denote the gene-gene network, let $P$ denote the bipartite network between genes and phenotypes, and let $Q$ denote the phenotype-phenotype network. In particular, $P=\left[\begin{array}{ll}P_{H s} & P_{S}\end{array}\right]$ is a composite of the gene-disease network of humans, written $P_{H s}$, and the gene-phenotype networks of other species, written $P_{S}$. Similarly,

$$
Q=\left[\begin{array}{cc}
Q_{H s} & 0 \\
0 & Q_{S}
\end{array}\right],
$$

where $Q_{H_{S}}$ is the similarity matrix of human diseases, and $Q_{S}$ is that of phenotypes of other species. In our experiments, we set $Q_{S}=0$, since we do not have information about similarity between phenotypes of other (non-human) species. The construction of the matrices $G, P$ and $Q_{H s}$ will be discussed in detail in the Methods section. We form a heterogeneous network over the gene and phenotype nodes, similar to RWRH (which we will review briefly in the Methods section). The adjacency matrix of the heterogeneous network may be written as:

$$
C=\left[\begin{array}{cc}
G & P \\
P^{\mathrm{T}} & Q
\end{array}\right],
$$

Recall the general formula for the truncated Katz similarity measure when specialized to the combined matrix $C$ : 


$$
S^{K a t z}(C)_{i j}=\sum_{l=1}^{k} \beta^{l}\left(C^{l}\right)_{i j} .
$$

From here on, we will drop the "truncated" and refer to this simply as the Katz measure. Note that for smaller values of $\beta$, higher order paths contribute much less. It has been shown that restricting the sum to a small $k$, i.e. a few higher order paths, works well in practice, in network link prediction and recommender systems[23]. Letting $k=3$, the block of the Katz score matrix $S^{\text {Katz }}(C)$ corresponding to similarities between gene nodes and human disease nodes, written $S_{H s}^{K a t z}(C)$, can be expressed as:

$$
\begin{aligned}
S_{H s}^{K a t z}(C) & =\beta P_{H s}+\beta^{2}\left(G P_{H s}+P_{H s} Q_{H s}\right) \\
& +\beta^{3}\left(P P^{T} P_{H s}+G^{2} P_{H s}+G P_{H s} Q_{H s}+P_{H s} Q_{H s}^{2}\right)
\end{aligned}
$$

where $P_{H s}$ and $Q_{H s}$ denote the gene-phenotype and phenotypephenotype networks of humans respectively. We use equation (5) to compute scores for the Katz method in our experiments.

In case of the drugs data set, we use the gene-drug network $D$, instead of $P_{H s}$ in equation (5). We do not have similarity information for drugs, and so we set $Q=0$ for experiments on the drug data set. Nonetheless, we use phenotype information from multiple species, in the composite matrix $P=\left[\begin{array}{ll}D & P_{S}\end{array}\right]$, in order to infer similarities between gene and drug nodes, where the matrix $D$ represents the known associations between genes and drugs (replacing $P_{H s}$ above in the gene-disease example).

\section{CATAPULT: A supervised approach to predicting associations}

The fixed choice of parameters involved in the Katz and random walk based approaches, as in equation (4), provides a reasonable initial approach. However, to improve performance we would like to learn the weights based on the heterogeneous network itself. That is, instead of using the exponential damping weights $\beta^{l}$, we can try to learn the relative importance of paths of different lengths. To this end, we frame the problem of predicting potential gene-phenotype associations as a supervised learning problem, in which we want to learn a classifier (or ranking) function whose input space consists of gene-phenotype pairs and output is a score for each gene-phenotype pair. In particular, by appropriately defining the feature space for gene-phenotype pairs, we will see that learning a classifier in the constructed feature space is tantamount to learning coefficients for Katz on the heterogeneous network computed as in equation (5).

For any given phenotype, it is very hard to verify that a gene is not associated in some way with the phenotype. Our learning strategy is therefore guided by the fact that absence of evidence is not evidence of absence. While a biological experiment can give clear evidence for the existence of a certain gene-phenotype association, a lack of evidence for a connection does not imply that such a connection does not exist. Biologists therefore tend to report positive associations between genes and phenotypes. However, the reported list of gene-phenotype associations is not exhaustive. Because negative associations rarely get reported, we treat all gene-phenotype pairs for which no positive association has been reported as unlabeled, with the prior assumption that most of them are in fact negative associations. Our data set therefore has the following two key characteristics:
1..For each phenotype, we only have a partial list of the associated genes. That is, we only know of positive associations; we do not have negative associations available to us.

2..There is a large number of unlabeled gene-phenotype pairs with the prior knowledge that most of them are, in fact, negative associations.

Classical supervised learning methods require both positive and negative examples, and therefore fall short in our case. PositiveUnlabeled learning (PU learning for short) methods are natural for this setting. The general idea of PU learning methods is to identify a set of negatives from the unlabeled examples and train a supervised classifier using the positives and the identified negatives. Liu et al.[24] study different ways of choosing negatives from unlabeled examples. Biologists believe that only a few of the large number of unobserved associations are likely to be positive. A random sample is likely to consist mostly of negatives, which suggests that we could randomly choose a set of examples and use the random sample as "negative" examples to train a supervised classifier. As the examples are not known to be negative, it may be helpful to allow the classifier to not heavily penalize the mistakes on "negatives" in the training phase. We therefore learn a biased support vector machine classifier using the positive associations and a random sample of unlabeled associations. Recently, Mordelet et al.[25] proposed ProDiGe that also uses a random sample of unlabeled examples as a negative sample to train a biased support vector machine against a set of known positives. The support vector machine is biased in the sense that false negatives (known positives classified as negatives) are penalized more heavily than the false positives ("negatives" classified as positives). The bias makes sense because the positive examples are known to be positive, while the negatives are arbitrary and hence false positives are not to be penalized too heavily. Note that, in principle, we could use any PU learning method (for instance, the weighted logistic regression model proposed in [26]) to obtain a classifier for gene-phenotype pairs.

Figure 2 demonstrates simple walk-based features derived from the heterogeneous network. Gene-phenotype pairs are represented using the walk-based features, and classified using a biased support vector machine in our CATAPULT algorithm.

Functional data outperforms protein-protein interactions

To see how the Katz and CATAPULT methods compare to the state-of-the-art, we measured their recovery of genes using a crossvalidation strategy similar to the one used by Mordelet and Vert [15], on two different data sets - gene-disease associations from the Online Mendelian Inheritance in Man (OMIM, [27]), and a recent drug-gene interaction data set [19]. These data sets can both be thought of as large collections of gene-trait pairs, either as gene-disease pairs for the OMIM data, or target-drug pairs for the drug data set.

We compared Katz and CAtaPUlt to four recent methods:

1..The recently proposed ProDiGe method [15], which is a support vector machine based method that calculates similarity scores for gene pairs using a wide variety of information sources including 21 different gene-gene functional interaction networks and phenotype similarities.

2..RWRH [8], which, like Katz uses walks on a heterogeneous gene-disease graph to prioritize genes. It differs from the Katz method chiefly in how the heterogeneous network is normalized. We discuss the relationship in more detail in Text S1.

3..We include PRINGE [7] for completeness, since it is the stateof-the-art to which both RWRH and ProDiGe were compared. 


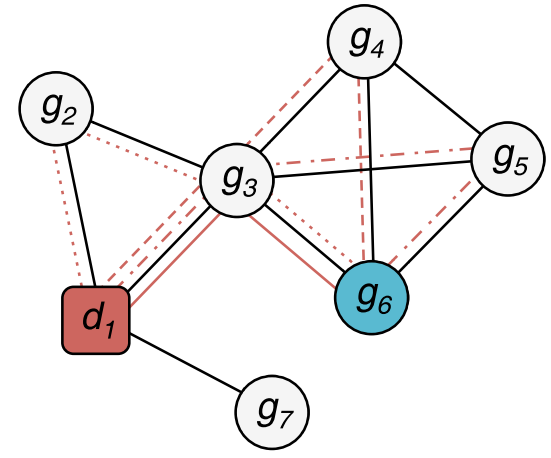

Figure 2. Katz features are derived by constructing walks of different kinds on the graph. In the figure above, the disease node $d_{1}$ is connected to the gene node $g_{6}$ by one walk of length 2 (solid red line) and three walks of length 3 (dotted, dashed and dashdotted red lines). This can be quickly calculated from the adjacency matrix $C$ of the graph: If $C_{i j}=1$ when there is a link between nodes $i$ and $j$, and 0 otherwise, the number of paths of length $n$ between genes $i$ and $j$ is $\left(C^{n}\right)_{i j}$. In the example above, $\left(C^{2}\right)_{16}=1$ and $\left(C^{3}\right)_{16}=3$.

doi:10.1371/journal.pone.0058977.g002

4..Finally, some recent work [18] has shown that simply by ranking based on the degree centrality of a gene (how often it interacts with other genes, or is involved in diseases) can be a very competitive ranking strategy. We therefore predict genes for diseases (or drugs) using a simple degree-based list, where all genes are ranked by how many diseases (drugs) they are known to be connected to, regardless of which disease (drug) the predictions are made for.

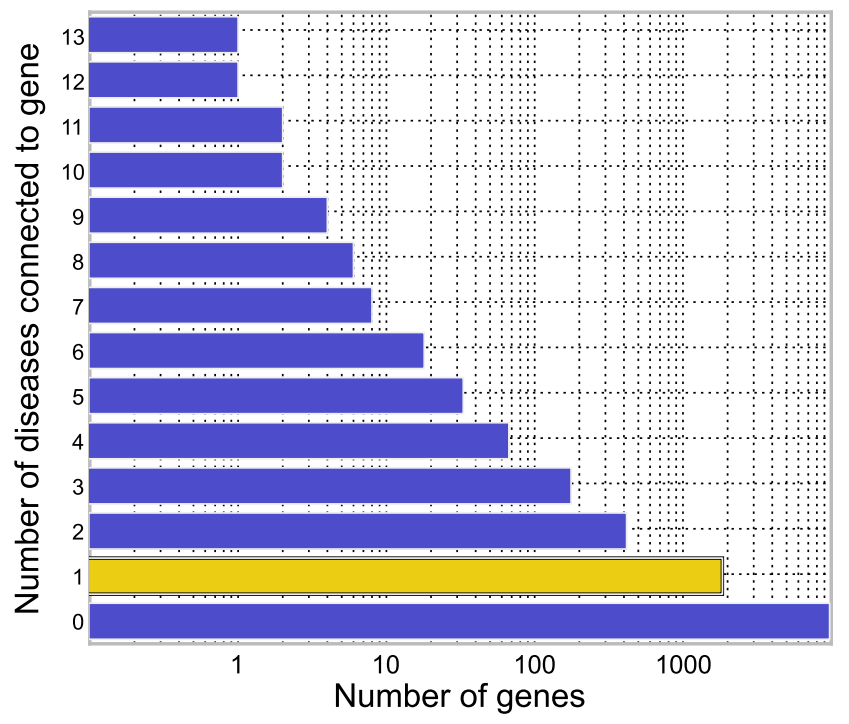

For cross-validation, we use the same testing framework as the one used by Mordelet and Vert [15]: split the known gene-trait pairs into three equally sized groups. We hide the associations in one group and run our methods on the remaining associations, repeating three times to ensure that each group is hidden exactly once. A clarification on the correctness of the cross-validation procedure while using heterogeneous information sources is in order: We do not create any gene-phenotype associations beyond those that are directly experimentally observed. We incorporate data from other species by orthology, but link it directly to the corresponding human gene, and do not create gene nodes for the orthologous species. Moreover, the only phenotype-phenotype linkages we include are across human disease pairs and are derived solely from MimMiner text mining of OMIM records [28] — there is no incorporation of gene sequence information whatsoever, only a measure of similarity of the clinical features of the diseases. Thus, there is no circularity created by homology in the data during cross-validation. For each trait in our data set, we order all the genes by how strongly the method predicts them to be associated with the trait. Finally, for every gene-trait pair $(g, t)$ in the hidden group we record the rank of the gene $g$ in the list associated with trait $t$. We use the cumulative distribution of the ranks as a measure for comparing the performances of different methods, i.e. the probability that the rank (at which hidden gene-trait pair is retrieved) is less than a threshold $k$. The motivation of using this performance measure is that a method that has a higher probability of recovering a true association in the top- $k$ predictions for a given disease is desired. Recent methods including ProDiGe[15] have adopted this performance measure for comparison.

The results are presented in Figure 3. Note that the vertical axis in the plots give the probability that a true gene association is

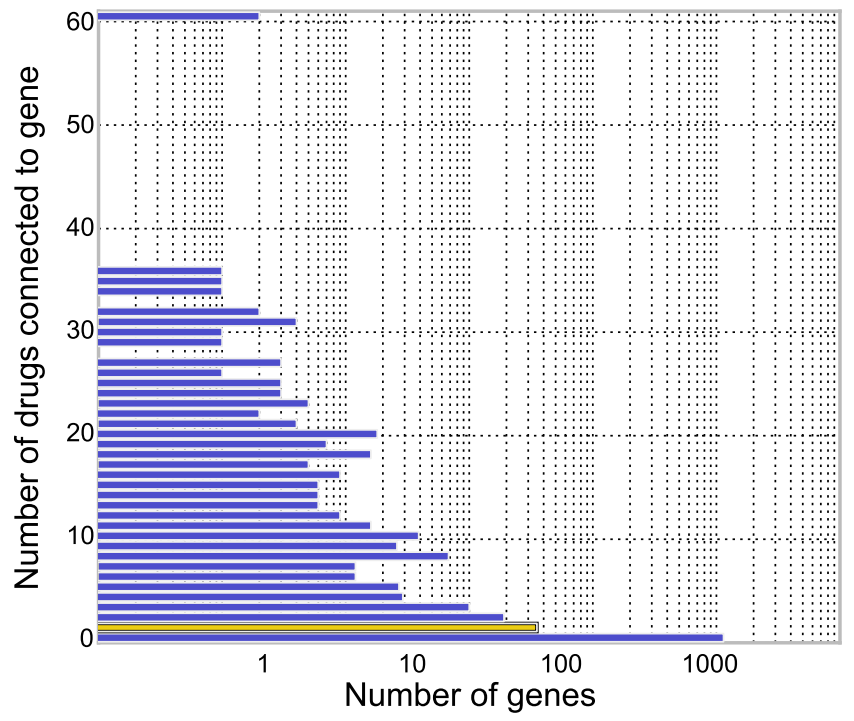

Figure 3. Empirical cumulative distribution function for the rank of the withheld gene under cross-validation. Left panel corresponds to evaluation of OMIM phenotypes, and the right corresponds to drug data. The vertical axis shows the probability that a true gene association is retrieved in the top- $k$ predictions for a disease. Katz and CATAPULT methods use all species information, and the HumanNet gene network. PRINCE and RWRH methods are implemented as proposed in [7] and [8] respectively, using the HPRD gene network. ProDiGe method is implemented as discussed in Methods section. CATAPULT (solid red) does much better across the data sets under this evaluation scheme. In general, the methods get high precision rates in case of the drug data. PRINCE method that does not allow walks through species phenotypes, and OMIM phenotypes in particular, performs much worse than other random-walk based methods. ProDiGe allows sharing of information between phenotypes using the similarities between OMIM phenotypes and performs reasonably well, whereas there is no such sharing possible in case of the drug data due to the absence of drug similarities. The simple degree-based method performs poorly in general. ProDiGe and PRINCE essentially use only the gene network information in case of the drug data.

doi:10.1371/journal.pone.0058977.g003 

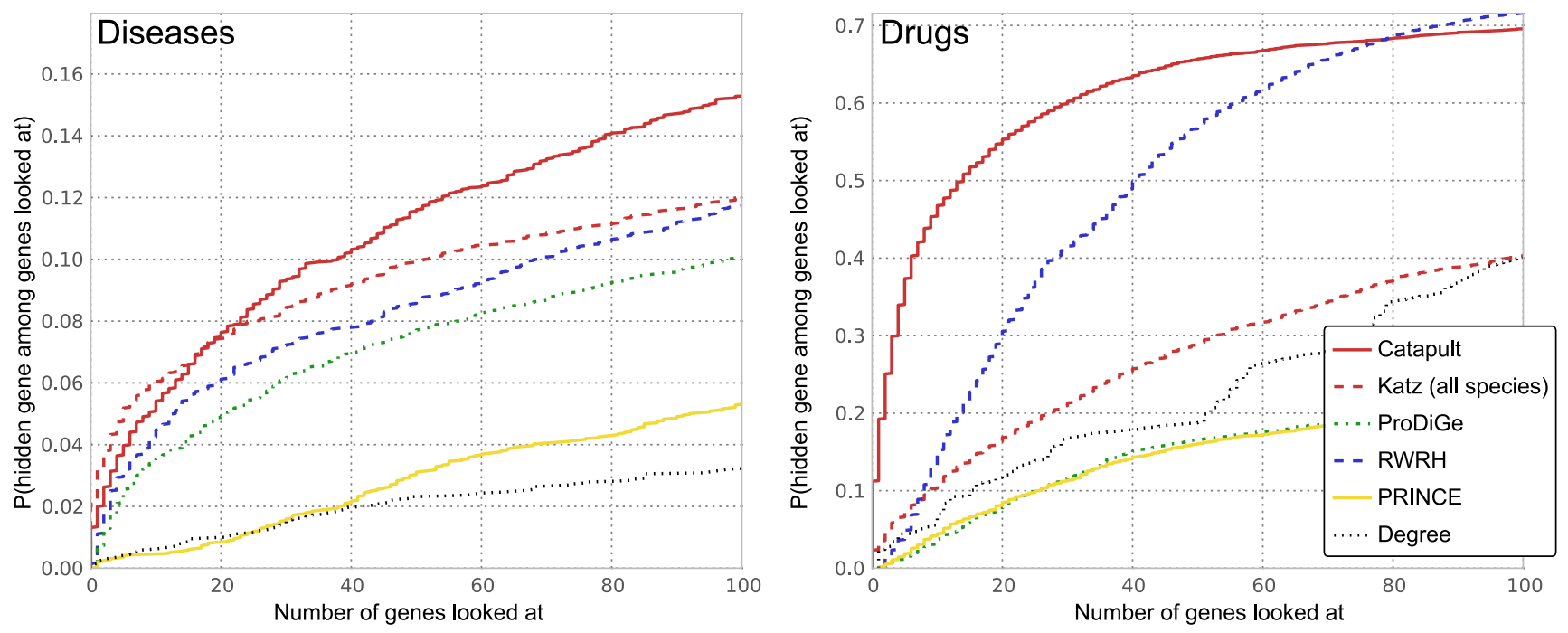

Figure 4. Comparison only using HumanNet. Empirical cumulative distribution function for the rank of the withheld gene under crossvalidation. Left panel corresponds to evaluation of OMIM phenotypes, and the right corresponds to drug data. The vertical axis shows the probability that a true gene association is retrieved in the top- $k$ predictions for a disease. Katz and CATAPULt methods use all species information, and all the methods use the HumanNet gene network. PRINCE and RWRH methods are implemented as proposed in [7] and [8] respectively, but using HumanNet. ProDiGe method is implemented as discussed in Methods section. Again, as in Figure 3, CATAPULt (solid red) does the best. An important observation to be made from the plots is that PRINCE and RWRH methods perform relatively much better than in Figure 3, where HPRD network was used. (Note that there is no change to the ProDiGe, Katz and CATAPult methods; they have identical settings as in Figure 3).

doi:10.1371/journal.pone.0058977.g004

recovered in the top- $k$ predictions for various $k$ values in the horizontal axis. For example, we observe that the Katz method has over $15 \%$ probability of recovering a true gene in the top- 100 predictions for a disease, whereas PRINCE is only $5 \%$ likely to retrieve a true association in the top- 100 predictions. Under this evaluation method, both Katz and Catapult, which make use of much more extensive data sets than the other methods, are quite likely to recover the hidden gene among the top 100 genes. As can be seen from Figure 3, Katz and CATAPULT perform better than any of the previously studied state-of-the-art gene-disease association methods for the OMIM data set. CATAPULT also performs well on the drug data set, ranking the hidden gene 14th or lower a remarkable $50 \%$ of the time. RWRH, which like Katz and CATAPULT is a walk based method that allows paths through the gene-disease (or, for the drug data set, gene-drug) network, also does quite well.

ProDiGe allows sharing of information between phenotypes using the similarities between OMIM phenotypes, and also integrates a wide variety of functional information in a supervised machine learning framework and performs reasonably well on the OMIM phenotypes. The PRINCE method, which allows some sharing of information between OMIM diseases that are phenotypically similar, performs worse than the other randomwalk based methods. Since we have no similarity information available for the drug data, ProDiGe and PRINCE essentially use only the gene similarity information in the drug data case. Notice that the simple degree-based method does the worst of all methods in case of OMIM phenotypes, which suggests that recommendations given by walk-based methods are more relevant and differ significantly from simple ranking by number of known associations.

To see if the improvement in performance of Katz and Catapult stems from the more extensive network used, or, in Catapult's case, the increased sophistication of the machine learning method, we evaluated network based RWRH and
PRINCE methods using the more extensive HumanNet network instead of the HPRD network originally used. As can be seen in Figure 4, Catapult still does better than the previous state-of-theart using this cross-validation framework, consistently in both the OMIM and drug data sets.

Precision-Recall measure. We also evaluate and compare the different methods using the more familiar precision and recall measures. Precision measures the fraction of true positives (genes) recovered in the top- $k$ predictions for a trait. Recall is the ratio of true positives recovered in the top- $k$ predictions for a trait to the total number of true positives in the hidden set. The plot of precision vs recall rates for different values of thresholds $k$ ranging $1 \leq k \leq 200$ is presented in Figure 5. Note that we are more interested in small values of $k$, similar to the results corresponding to the rank cdf measure presented in Figures 3,4 and 6. Our experimental setup is identical to that in Figure 4, i.e. using HumanNet for all the relevant methods. The comparisons on both OMIM and drug data sets observed from Figure 5 are identical to Figure 4. In particular, CATAPULT performs much better in case of the drug data, and is competitive to Katz and RWRH methods at very small values of $k$ (in the range $1 \leq k \leq 20$ ) and performs much better outside the regime, in case of the OMIM data. Note that we observe identical behavior for CATAPULT in the left panel of Figure 4. The performance of the other methods are similarly consistent across the two performance measures.

Focusing on the gene linkage neighborhood. The results so far are on measuring how well a candidate gene can be predicted genome wide. Another common scenario is where a linkage interval is known for a disease, but the causal disease linked gene has not been identified. To simulate this setting, we use an approach similar to the one taken in [7]. For each known genedisease pair $(g, t)$, we construct a simulated linkage interval by taking all genes within 10 million basepairs from either end of the gene $g$ (containing a median of 84 genes), and record the rank at which gene $g$ is predicted for trait $t$ when $g$ is masked. As can be seen in Figure 7, Catapult again performs the best. 

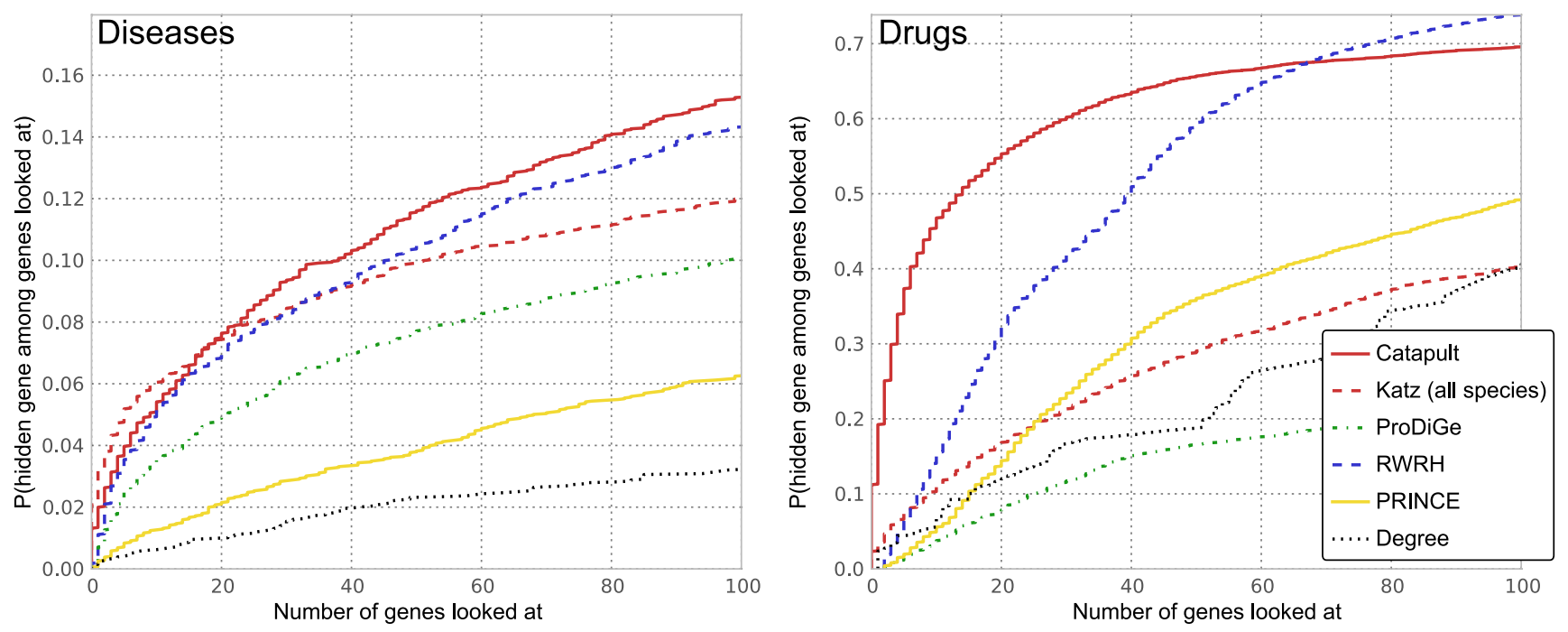

Figure 5. Precision-Recall curves for three-fold cross validation. Left panel corresponds to evaluation of OMIM phenotypes, and the right corresponds to drug data. The vertical axis shows the precision rate, i.e. fraction of true positives in the top- $k$ predictions. The horizontal axis shows the recall rate, i.e. ratio of true positives recovered in the top- $k$ predictions to the total number of positives for a phenotype (or a drug) in the hidden set. The plots show precision-recall values at various thresholds $k$, in the range $1 \leq k \leq 200$ and the value at a given $k$ is averaged over all the phenotypes (drugs). The plots use the same experimental setup as in Figure 4, and we observe that the comparisons illustrated by precision-recall measure are consistent with the rank cdf measure in Figure 4.

doi:10.1371/journal.pone.0058977.g005

When do GATAPULt and GBA methods fail? It is important to know if there is a set of phenotypes for which Catapult, and other network-based GBA methods, do not perform well. To understand the same, we looked at the phenotypes for which CATAPULT attained the poorest (average) recall rate. In particular, we ordered the phenotypes by the (mean) recall rate for the hidden genes (in three-fold validation). We find that the bottom-most phenotypes in the ordering are precisely the ones for which there is only one known gene. Note that the training data for these phenotypes did not have the known gene. The only information for the phenotypes comes from the phenotype-phenotype similarities. However, there are some phenotypes for which even phenotype similarities are not known. In such cases, all GBA or networkbased methods will fail. The results are presented in Figure 8 (left panel). We observe that all network-based methods perform poorly. Nonetheless, we observe a gradation in the performances of different methods, and Catapult does slightly better. The difference in performance is not surprising given that some methods use the heterogeneous network fully ( CATAPULT and Katz) but others only partially (ProDiGe, PRINCE and RWRH). All the methods do substantially better on phenotypes with more than one known gene (right panel). A qualitative analysis of the methods discussed next, however, shows that the boost in performance may not necessarily reflect that the predictions made by the methods are pertinent to the phenotypes. This connection between the size of sets and how easy they are to predict has also been observed in the context of GO annotations, see for instance [29].

\section{Top predictions by supervised methods are enriched for highly connected genes}

To get a qualitative view of how the connectedness of genes influences the rankings, we plotted the degree distribution of the genes in the OMIM and the drug data sets in Figure 9, and compared the results with the list of top candidates from Catapult (see Table 1) and Katz (see Table 2).
The results for CATAPULT all seem very reasonable, from a biological standpoint. For example, Catapult identifies APOE, which even though is not linked to "Susceptibility to Alzheimer's disease" OMIM record (MIM:104300), is well known to be associated with Alzheimer's disease and is associated with two other OMIM records involving Alzheimer's (MIM:104310 and MIM:606889). BRCA1 is associated with "Breast-ovarian cancer, familial 1" (MIM:604370), not the record we show in Table 1 ("Breast cancer, susceptibility to", MIM:114480), even so, it is ranked very highly among the candidate genes for breast cancer. Many of the other candidate genes listed are similarly very likely to be involved in the etiology of the diseases, like TP53 and KRAS for many cancers. Indeed, what might be the most surprising about the results is how completely unsurprising they seem. Furthermore, there is a very high degree of overlap between the top predictions. Indeed, almost all the top 10 candidate genes for the eight diseases shown are shared between at least two of the eight diseases for Catapult. Moreover, when studying the results for the same diseases for ProDiGe, given in [15], we see the same pattern as we see for CATAPULT - a strong enrichment for genes that are already known to be associated with many diseases. For example, EGFR is predicted as a top ten candidate gene for gastric cancer by ProDiGe, as well as by Catapult (Table 1) and the Katz method (Table 2). In case of Alzheimer's disease, our methods and ProDiGe all predict $A P O E$ in the top ten.

However, the top predictions for both Catapult and ProDiGe seem to be governed more by what method is being tested than by what disease is being studied. For example, ProDiGe ranks EXT1 in the top ten for six out of the eight diseases studied, and Catapult ranks TP53 in the top ten for five of the diseases. In contrast, the results for the Katz measure (Table 2) exhibit a much lower degree of overlap between the top predictions. There is still a certain number of predictions shared, particularly between the different cancers and insulin resistance (type 2 diabetes). However, there is a good reason to believe that these shared genes actually reflect a common etiology, since epidemiological studies have 
shown a connection between the type 2 diabetes and cancers, in particular breast and colorectal cancer [30]. Overall, the predictions seem to reflect the relevance of a gene to the specific disease more than the overall likelihood that a gene is associated with any disease.

For example, many of the top ranked genes for Alzheimer's disease are related to amyloid precursor protein, $A P P$, in various ways, such as APLP2 and APLP1, which are homologs of APP, $C T S B$, also known as amyloid precursor protein secretase, LRP1, which is necessary for clearance of $A P P$ plaques, and $A P O E$, apolipoprotein E. A recent review of the role $A P P$ and its interaction partners play in Alzheimer's disease can be found in [31]. CAV1 has also recently been studied in relation to APP and Alzheimer's disease [32]. Another interesting candidate Alzheimer's disease is BDKRB2, bradykinin receptor B2. BDKRB2 is highly expressed in the central nervous system according to the Human Protein Atlas, and modulation of BKRB2 results in a cellular state highly enriched for amyloid $\beta$ peptide in a skin fibroblast cell line from a patient with early onset familial Alzheimer's disease [33].

We see a similar pattern with more specific predictions that still seem well supported by the literature for most of the other diseases in Table 2. For example, the association of MYH11 with leukemia, through inversion of a region on chromosome 16 and the formation of a $C B F B-M Y H 11$ chimera, is well known and was first identified in [34]. However, it is not associated with the OMIM record shown in Table 2. For schizophrenia, the top ranked candidate gene, $D R D 2$, is well known to be associated with schizophrenia (under MIM:126450), and a recent study has highlighted a potential role for ADRA2B in schizophrenia [35].

\section{Validation on singletons highlights methods that detect novelty}

The cross-validation evaluation shown in Figures 3 and 4 clearly shows that CATAPULT is better at recapitulating the genes known to be involved in a disease than any of the other methods. However, recapitulation of previously known results is rarely the goal in biology. We therefore seek a measure that would reflect how suited a method is for correctly identifying associations between diseases and genes that are less well studied.

There are two ways one could envision for doing this in a crossvalidation framework - either one could hide all associations between a given gene and diseases, thereby hoping to put it on equal footing with genes still unstudied, or one restricts the crossvalidation to genes that are only associated with a single phenotype. There are clear advantages to both approaches. The former approach allows us to do validation on a larger set, namely all known gene-disease associations, and thereby reach stronger statistical strength. The latter approach has more subtle, but in our opinion greater, advantage. The biases that favor already well

Table 1. Top predictions for CATAPULT.

\begin{tabular}{|c|c|c|c|}
\hline Leukemia & Alzheimer disease & Insulin resistance & Prostate cancer \\
\hline MIM:601626 & MIM:104300 & MIM:125853 & MIM:176807 \\
\hline FGFR3 (2261) & ACE2 (59272) & INSR (3643) & TP53 (7157) \\
\hline FGFR2 (2263) & COL1A1 (1277) & INS (3630) & RB1 (5925) \\
\hline KRAS (3845) & COL1A2 (1278) & PTEN (5728) & CTNNB1 (1499) \\
\hline TP53 (7157) & KRAS (3845) & TP53 (7157) & BRCA1 (672) \\
\hline EGFR (1956) & EGFR (1956) & CTNNB1 (1499) & KRAS (3845) \\
\hline FGFR1 (2260) & TP53 (7157) & KRAS (3845) & PIK3CA (5290) \\
\hline PTPN11 (5781) & $A G T$ (183) & AKT1 (207) & AKT1 (207) \\
\hline CTNNB1 (1499) & PLAT (5327) & CREBBP (1387) & INSR (3643) \\
\hline INSR (3643) & APOE (348) & EGFR (1956) & NRAS (4893) \\
\hline CREBBP (1387) & PTGS2 (5743) & PIK3CA (5290) & RAD51 (5888) \\
\hline Schizophrenia & Breast cancer & Gastric cancer & Colorectal cancer \\
\hline MIM:181500 & MIM:114480 & MIM:137215 & MIM:114500 \\
\hline$B D N F(627)$ & PTEN (5728) & FGFR3 (2261) & KRAS (3845) \\
\hline NRG1 (3084) & $R B 1$ (5925) & FGFR1 (2260) & PTEN (5728) \\
\hline CBS (875) & NRAS (4893) & NRAS (4893) & CTNNB1 (1499) \\
\hline NOS2 (4843) & BRCA1 (672) & HRAS (3265) & HRAS (3265) \\
\hline MTR (4548) & HRAS (3265) & EGFR (1956) & CREBBP (1387) \\
\hline HTR2C (3358) & INSR (3643) & ERBB3 (2065) & RB1 (5925) \\
\hline HTR2B (3357) & CTNNB1 (1499) & CTNNB1 (1499) & FGFR3 (2261) \\
\hline SLC6A4 (6532) & EGFR (1956) & BRAF (673) & INSR (3643) \\
\hline FGFR2 (2263) & FGFR3 (2261) & PTEN (5728) & EGFR (1956) \\
\hline MAT1A (4143) & FGFR2 (2263) & TP53 (7157) & FGFR2 (2263) \\
\hline
\end{tabular}

Top 10 predictions not in the training set by CATAPULT for the eight OMIM phenotypes with the highest number of gene associations. Any gene which is among the top 10 candidates for more than one disease is marked in bold. CATAPULT does make a great number of very reasonable predictions as observed below. For example, it seems quite likely that both insulin receptor (INSR, 3643) and insulin (INS, 3630) should be associated with insulin resistance, and that many growth factor receptors have been associated with various cancers.

doi:10.1371/journal.pone.0058977.t001 
Table 2. Top predictions for the Katz measure.

\begin{tabular}{|c|c|c|c|}
\hline Leukemia & Alzheimer disease & Insulin resistance & Prostate cancer \\
\hline MIM:601626 & MIM:104300 & MIM:125853 & MIM:176807 \\
\hline IL3 (3562) & APLP2 (334) & INS (3630) & BRCA1 (672) \\
\hline SOCS1 (8651) & HSPA8 (3312) & AKT1 (207) & TP53 (7157) \\
\hline GRB2 (2885) & CTSB (1508) & INSR (3643) & RAD51 (5888) \\
\hline NOP2 (4839) & LRP1 (4035) & GRB2 (2885) & EGFR (1956) \\
\hline CSF2RB (1439) & NID1 (4811) & IGF1R (3480) & ATM (472) \\
\hline PPM1L (151742) & APOE (348) & CTNNB1 (1499) & AKT1 (207) \\
\hline PTPN6 (5777) & BDKRB2 (624) & CREBBP (1387) & $\operatorname{MAX}(4149)$ \\
\hline MYH11 (4629) & PLAUR (5329) & PIK3CA (5290) & CDK1 (983) \\
\hline PPM1E (22843) & APLP1 (333) & TYK2 (7297) & PIK3CA (5290) \\
\hline PPM1B (5495) & CAV1 (857) & GPD1 (2819) & CSNK2A1 (1457) \\
\hline Schizophrenia & Breast cancer & Gastric cancer & Colorectal cancer \\
\hline MIM:181500 & MIM:114480 & MIM:137215 & MIM:114500 \\
\hline DRD2 (1813) & BRCA1 (672) & GRB2 (2885) & PTEN (5728) \\
\hline$A H C Y$ (191) & IRS1 (3667) & EGFR (1956) & CTNNB1 (1499) \\
\hline$A D R A 2 B(151)$ & MRE11A (4361) & NRAS (4893) & CDK1 (983) \\
\hline XRN2 (22803) & INSR (3643) & IRS1 (3667) & GSK3B (2932) \\
\hline MAT1A (4143) & CHEK1 (1111) & MAPK1 (5594) & $C D C 20$ (991) \\
\hline MAT2A (4144) & ATR (545) & PTPN11 (5781) & PCNA (5111) \\
\hline CHI3L2 (1117) & PTEN (5728) & HRAS (3265) & $E G F(1950)$ \\
\hline TSNAX (7257) & MAPK1 (5594) & MAP2K2 (5605) & PTTG1 (9232) \\
\hline$D D C(1644)$ & MAPK3 (5595) & MAP2K1 (5604) & IGF1R (3480) \\
\hline MAOB (4129) & UBE2I (7329) & SOS1 (6654) & FOXO3 (2309) \\
\hline
\end{tabular}

Top 10 predictions not in the training set by Katz for the same eight OMIM phenotypes as in Table 1 . Any gene which is among the top 10 candidates for more than one disease is marked in bold. The Katz method shows a weaker link between the number of diseases previously associated with a gene and its presence in the list, while still giving a number of very likely candidates.

doi:10.1371/journal.pone.0058977.t002

studied genes are not only present in the gene-disease association data, but also in the data that we use for constructing the networks and the model species data sets. This gives rise to small differences between genes that have been the well studied genes and the poorly characterized genes. By only looking at the least studied genes in our data set for which we do have known gene-disease associations, we can minimize the risk that any signal that we detect is merely some general characteristic of well studied genes, and instead evaluate how well a method can detect truly novel gene-disease associations.

We tested all the methods using cross-validation restricted to genes with only a single disease (or drug) association (which we call singletons, shown in yellow in Figure 9). The results of this crossvalidation are presented in Figure 6. CAtapult still does much better than ProDiGe (the only other supervised method) but does worse than the unsupervised methods, in contrast to Figure 4 (that uses the same setting for all the methods). The PRINCE and ProDiGe methods are consistent with (and sometimes perform slightly better than) the three-fold cross-validation evaluation. RWRH and the Katz measure perform better than the supervised learning methods ProDiGe and CATAPULT in this evaluation sheme. The fact that PRINCE performs so well on singletons when evaluated on drug data is surprising, given that the only information it uses is the HumanNet gene network. Simpler random-walk based methods in general perform better than the supervised counterparts, and do so consistently in two completely distinct data sets. Furthermore, we find that the qualitative results of the methods (Tables 1 and 2) indicate that the supervised Catapult tends to emphasize the same "common" genes much more than the Katz measure does, which is consistent with the difference in performance we observe between the cross-validation on the full set of gene-disease associations and cross-validation restricted to the singleton genes.

The differences in performance between the full test set and the singletons raise the question of what we really are trying to do when we predict gene-phenotype associations. Ultimately, the correct evaluation criterion for gene-phenotype association predictions must be successful laboratory confirmation of the predictions. However, lacking that, we often resort to different cross-validation schemes to measure how well a method does. We have here shown that even quite modest changes in the evaluation scheme can alter the relative performance of the methods tested. In the case of CAtapult and the Katz measure, this is likely because the tendency of "common" genes to be involved in diseases in general is a property that the supervised CATAPUlt can easily detect and make use of, which strongly boosts its performance on the full data set but does nothing for its performance on the singleton genes. By actively restricting the use of features that are characteristic of "common" genes (For instance by the restricting the maximum allowed path length), we can counteract this tendency at the cost of performance in the full cross-validation (data not shown). 

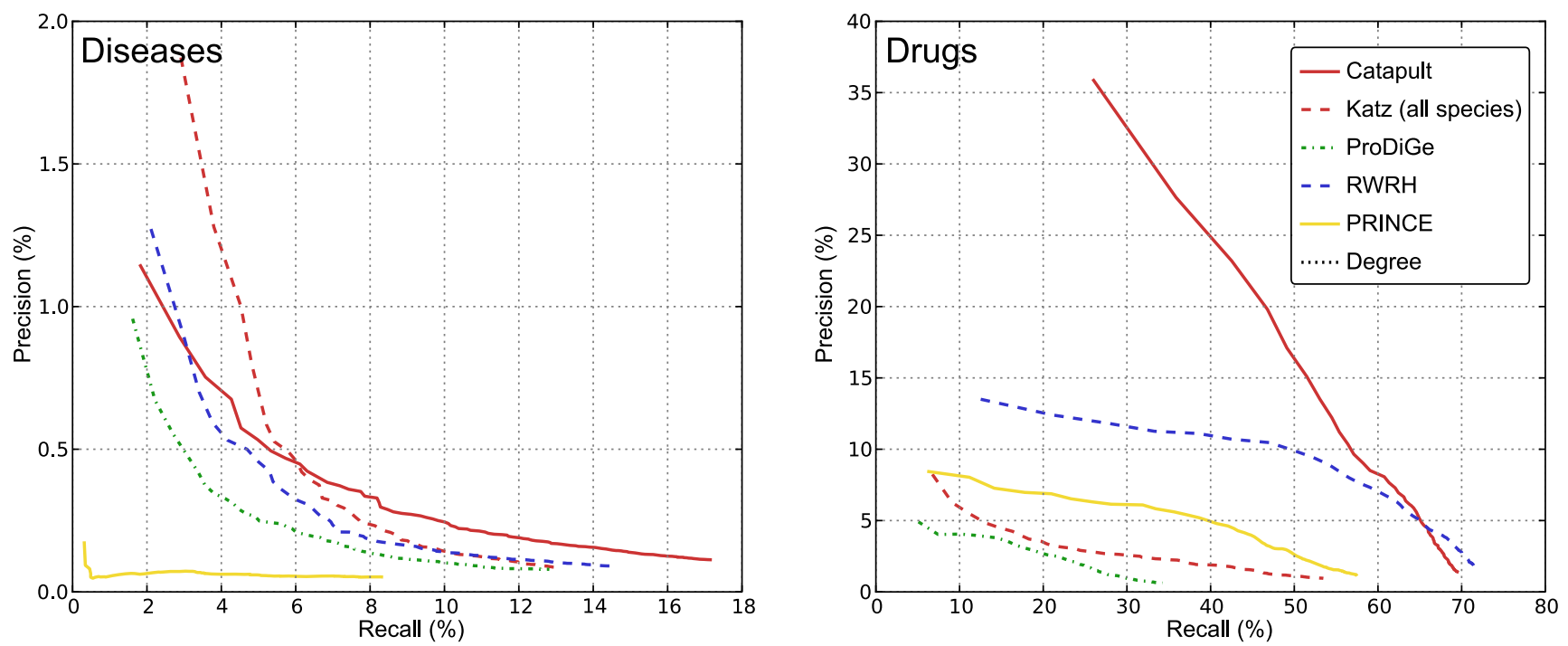

Figure 6. Empirical cumulative distribution function for the rank of withheld singleton genes. Left panel corresponds to evaluation of OMIM phenotypes, and the right corresponds to drug data. The vertical axis shows the probability that a true gene association is retrieved in the top$k$ predictions for a disease. The Katz and CATAPULt methods use all species information, and all the methods use the HumanNet gene network. PRINCE and RWRH are implemented as proposed in [7] and [8] respectively, but using the HumanNet gene network. The ProDiGe method is implemented as discussed in the Methods section. We have not included the degree based list from Figure 4, since all the singleton genes are always given degree 0 during cross-validation. CATAPULt (solid red) does much better than ProDiGe (the only other supervised method) but does worse compared to walk-based methods than in Figure 4 (that uses the same setting for all the methods). PRINCE and ProDiGe are consistent with (and sometimes perform slightly better than) the full cross-validation evaluation. RWRH and the Katz measure perform better than the supervised learning methods ProDiGe and CATAPULT in this evaluation scheme. The fact that PRINCE performs so well on singletons in the drug data case is surprising, given that the only information it uses is the HumanNet gene network. doi:10.1371/journal.pone.0058977.g006

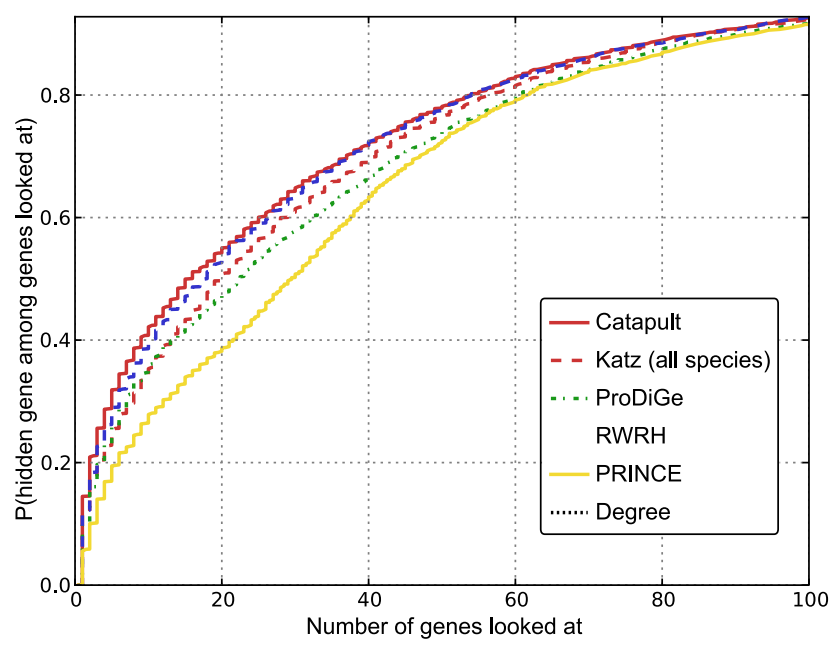

Figure 7. Empirical cumulative distribution function for the rank of withheld genes from OMIM phenotypes, restricted to genes in a small linkage neighborhood of the withheld genes. The vertical axis shows the probability that a true gene association is retrieved in the top- $k$ predictions for a disease. The Katz and CATAPULT methods use all species information, and all the methods use the HumanNet gene network. PRINCE and RWRH are implemented as proposed in [7] and [8] respectively but using the HumanNet gene network. The ProDiGe method is implemented as discussed in the Methods section. We observe that CATAPULT performs the best. RWRH and Katz methods are competitive as well. The results are consistent with our observations from Figure 4.

doi:10.1371/journal.pone.0058977.g007

\section{Conclusions}

We have proposed two methods for inferring gene-phenotype associations, Katz and CATAPULt. Katz is motivated by social network link prediction and CATAPULT is a supervised extension to Katz which learns the weights for walks that have different lengths and that involve different kinds of data. While CAatapult significantly outperforms other state-of-the-art gene-phenotype association methods using a conventional cross-validation evaluation strategy, such a cross-validation strategy does not necessarily reflect the properties of a scenario in which one wants to predict novel gene-phenotype associations involving less studied genes.

To address such cases, we propose a cross-validation approach restricted to relatively little studied genes. In this framework the Katz method and the related RWRH and PRINCE methods do better than the supervised methods, indicating that if the objective is to find new gene-disease or gene-drug associations involving genes not yet well studied, these approaches are more appropriate.

We therefore conclude that comparisons of gene-phenotype methods do not necessarily lead to a simple ordering from best to worst. If the goal of a researcher is to find new directions for research or find previously unknown biology, she might not want to use methods that perform the best in a conventional crossvalidation framework. For instance, she might prefer a method like the Katz measure, which does better when tested on genes only associated with a single disease, to a method like CATAPULT, which emphasizes genes that are important in general. In the future, it is therefore important that descriptions of new gene-phenotype association methods include a careful discussion on how the method is intended to be used. 

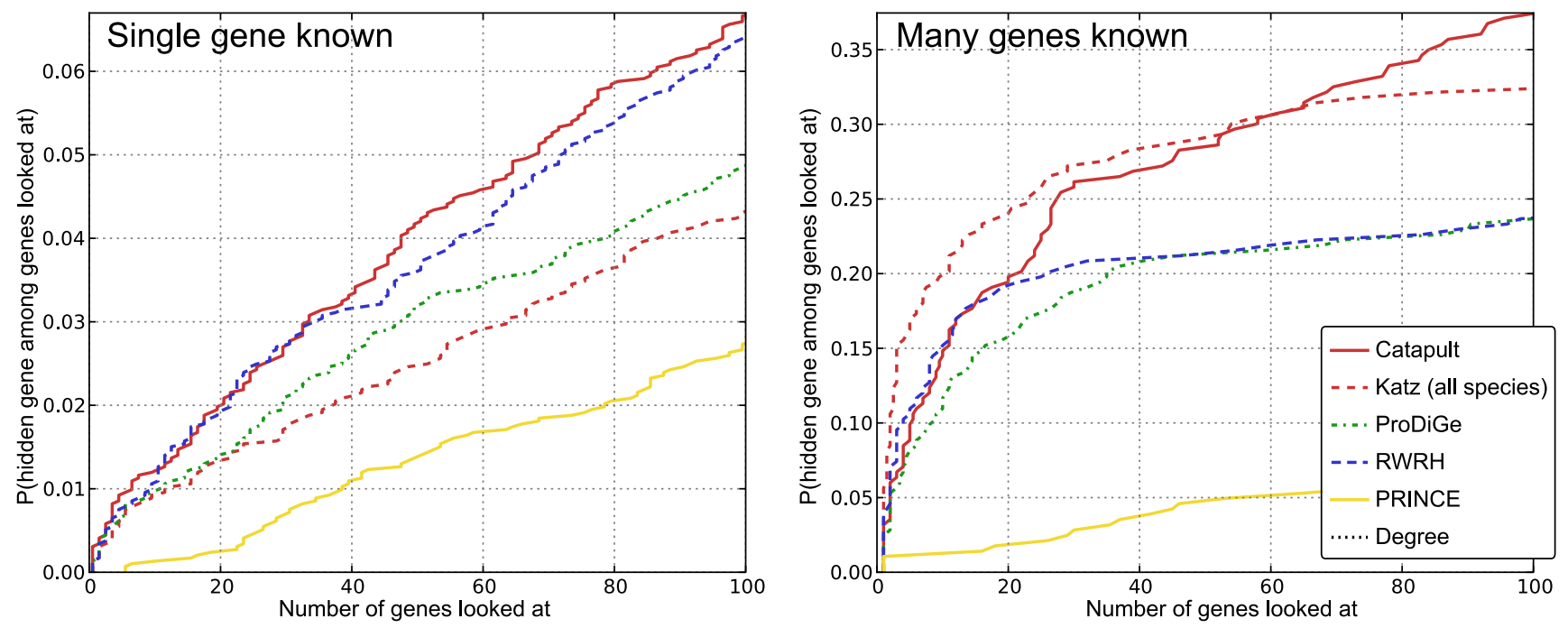

Figure 8. Empirical cumulative distribution function for the rank of withheld genes from OMIM phenotypes with single known gene (left panel) and more than one known gene (right panel). The vertical axis shows the probability that a true gene association is retrieved in the top- $k$ predictions for a disease. The Katz and CATAPULT methods use all species information. PRINCE and RWRH are implemented as proposed in [7] and [8] respectively, using HPRD network. The ProDiGe method is implemented as discussed in the Methods section. In case of phenotypes with only one known gene (left panel), the only information is the phenotype-phenotype similarity. From the left panel, we note that all network-based methods perform poorly. Nonetheless, we observe a gradation in the performances of different methods, and that CATAPULT does slightly better. All the methods do substantially better on phenotypes with more than one known gene (right panel).

doi:10.1371/journal.pone.0058977.g008

\section{Materials and Methods}

Gene Networks

We use two sources of gene-gene interactions in our experiments.

1..HumanNet [14]: A large-scale functional gene network which incorporates multiple data sets, including mRNA expression, protein-protein interactions, protein complex data, and comparative genomics (but not disease or phenotype data). HumanNet contains 21 different data sources, which are combined into one integrated network using a regularized regression scheme trained on GO pathways. The edges in the network have non-negative edge weights, and there are 733,836 edges with non-zero weights.

2..HPRD network [36]: Most of the published work on predicting gene-disease associations [5-8,37] use the HPRD network. The network data was downloaded from [4]. The edges in the HPRD network are unweighted, and the network is much sparser than HumanNet. In particular, the HPRD network has 56,661 associations compared to 733,836 (weighted) associations for HumanNet.
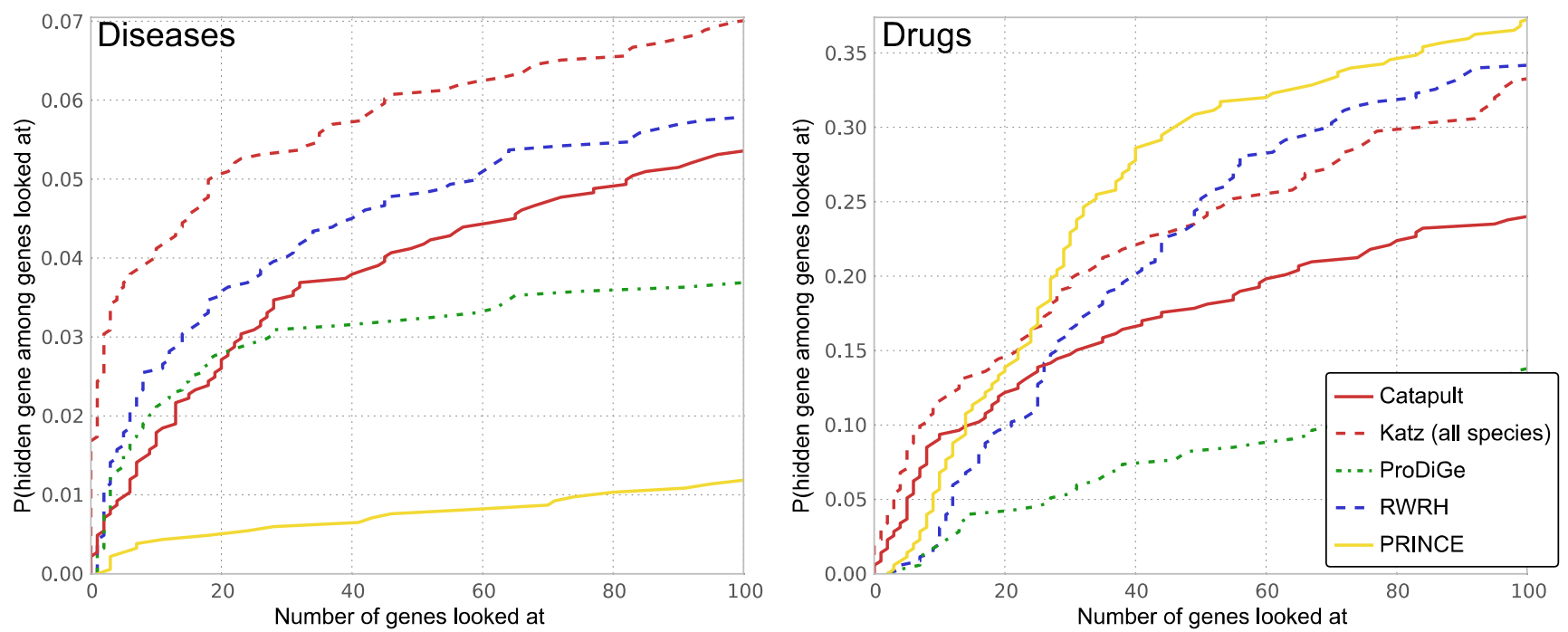

Figure 9. Distribution of the number of known genes in OMIM diseases (left) and drugs (right). The bar corresponding to the genes on which we did the singleton validation is shown in yellow.

doi:10.1371/journal.pone.0058977.g009 


\section{Phenotypes from other (non-human) species}

We collected gene-phenotype associations from literature and public databases for eight different (non-human) species: plant (Arabidopsis thaliana, from TAIR [38]), worm (Caenorhabditis elegans from WormBase [39,40]), fruit fly (Drosophila melanogaster from FlyBase [41]), mouse (Mus musculus from MGD [42]), yeast (Saccharomyces cerevisiae [10,43-45]), Escherichia coli [46], zebrafish (Danio rerio from ZFIN [47]), and chicken (Gallus gallus from GEISHA [48]). We determined orthology relationships between genes in model species and human using INPARANOID [49]. Detailed description on the extraction of most datasets can be found in [16] and the resulting dataset has been summarized in Table 3 .

E. coli phenotypes were obtained from the file 'coli_FinalData2.txt' on May 20, 2011 [46]; we sorted each gene's phenomic profile by score, taking both the top and bottom forty conditions and assigning them to the gene. Thus, we considered each condition to be a phenotype, and the genes associated with that phenotype were those genes whose growth was most affected (either positively or negatively) in the corresponding condition. As a proxy for chicken phenotypes, tissue specific mRNA expression patterns were derived from GEISHA in situ hybridization annotations, which were kindly provided in XML format on June 24, 2011. Genes were sorted into multiple bins by stage, by location, and by location and stage together. If there were more than fifty genes in a specific location and more than three at a specific stage at that location, a new phenotype was created ("anatomical location at stage $x$ "); regardless, each location became a phenotype. Worm phenotypes [40] were divided into two datasets, 'green-broad' and 'green-specific', based on the broad and specific phenotypes. GO biological processes from TAIR and ZFIN were processed in the same manner. We kept only those annotations with evidence codes IMP, IDA, IPI, IGI, TAS, NAS, IC, and IEP. For TAIR, we used 'ATH_GO_GOSLIM.txt', downloaded on August 23rd, 2010; and for ZFIN, we obtained GO biological processes from geneontology.org ('gene_association.zfin.gz') on April 26th, 2011.

\section{Evaluation data}

We perform experiments on two types of data sources:

- OMIM Phenotypes: We obtained new OMIM data from the Online Mendelian Inheritance in Man (OMIM) project

Table 3. Species used.

\begin{tabular}{llll}
\hline & & & \\
\hline Index & Species & \# Phenotypes & \# Associations \\
\hline 1 & Human $(H s)$ & 3,209 & 3,954 \\
2 & Plant $(A t)$ & 1,137 & 12,010 \\
3 & Worm $(C e)$ & 744 & 30,519 \\
4 & Fly $(\mathrm{Dm})$ & 2,503 & 68,525 \\
5 & Zebrafish $(\mathrm{Dr})$ & 1,143 & 4,500 \\
6 & E.coli $(\mathrm{EC})$ & 324 & 72,846 \\
7 & Chicken $(\mathrm{Gg})$ & 1,188 & 22,150 \\
8 & Mouse $(\mathrm{Mm})$ & 4,662 & 75,199 \\
9 & Yeast $(\mathrm{Sc})$ & 1,243 & 73,284 \\
\hline
\end{tabular}

Different species used for inferring gene-phenotype associations in the proposed methods Katz and CATAPULT, and sizes of the gene-phenotype networks for the species, restricted to orthologs of human genes. The total number of human genes with any kind of phenotype annotation is 12331 . doi:10.1371/journal.pone.0058977.t003
[27] on August 11, 2011. OMIM phenotypes have become the standard data set for the evaluation of prediction of genedisease associations [5-8,15,37]. All the compared methods use similarities between phenotypes [28] to form the (weighted) phenotype-phenotype network $Q_{H s}$.

- Drug data: This includes four benchmark data sets of DrugTarget interactions in humans involving enzymes, ion channels, G-protein-coupled receptors (GPCRs) and nuclear receptors, first studied in [50]. Refer to Table 4 for statistics on the data sets. The data sets were made available by [19] and downloaded from [51].

\section{Problem setup and Notation}

Let $\mathcal{G}$ denote the set of human genes and for each species $i \in \mathcal{S}=\{H s, A t, C e, D m, D r, E c, G g, M m, S c\}$, let $\mathcal{P}_{i}$ denote the set of phenotypes for the species. Refer Table 3 for descriptions of the species and a summary of the data sets. Also, let $\mathcal{D}$ denote the set of drugs (i.e. the four benchmark data sets mentioned in Table 4). For each species $i \in \mathcal{S}$, we constructed a gene-phenotype association matrix $P_{i} \in \mathbb{R}^{|\mathcal{G}| \times\left|\mathcal{P}_{i}\right|}$, such that $\left(P_{i}\right)_{g p}=1$ if gene $g$ is associated with phenotype $p$ or 0 otherwise. For methods using multiple species, we used $P_{S}=\left[\begin{array}{lllll}P_{A t} & P_{C e} & P_{D m} & \ldots & P_{S c}\end{array}\right]$ and recall that $P=\left[\begin{array}{ll}P_{H s} & P_{S}\end{array}\right]$ in equation (5). Similarly, we constructed a druggene interaction matrix $D$ using drugs data where $D_{g d}=1$ if gene $g$ is known to be associated with drug $d$ (note that $d$ can be one of enzymes, ion channels, GPCRs or nuclear receptors) and $D_{g d}=0$ otherwise. Using the two types of gene-gene interaction data HPRD and HumanNet, we constructed matrices $G^{H P R D} \in\{0,1\}^{|\mathcal{G}| \times|\mathcal{G}|}$, and $G^{\text {HumanNet }} \in \mathbb{R}^{|\mathcal{G}| \times|\mathcal{G}|}$ respectively. We constructed a phenotype-phenotype network $Q_{H s} \in \mathbb{R}^{\left|\mathcal{P}_{H s}\right| \times\left|\mathcal{P}_{H s}\right|}$ (i.e. corresponding to humans) using OMIM phenotype similarities [28]. For experiments with drug data, we did not have access to any such similarity score for drug pairs, so we set the drug-drug network to 0 . The same is the case for other species data as well, and we set the corresponding entries in $Q$ to be 0 , both for the experiments with OMIM and for the drug data. Following the approach by Vanunu et al. [7], we apply a logistic transformation to the similarities $Q_{H s}$, i.e. $L(x)=\frac{1}{1+\exp (c x+d)}$ where $x$ represents an entry of $Q_{H s}$. For setting $c$ and $d$, see [7]. We use the transformed $Q_{H s}$ network in all our experiments.

The GATAPULT algorithm. CATAPUlt uses a biased SVM framework to classify gene-phenotype pairs of humans with a single training phase, thereby making use of the relation between different phenotypes. Recent work [25] uses the bagging technique to obtain an aggregate classifier using positive and unlabeled examples. In this approach, one draws a random bootstrap sample of a few unlabeled examples from the set of all unlabeled examples and trains a classifier treating the bootstrap sample as negatives along with the positive examples. Bagging helps to reduce the variance in the classifier that is induced due to the randomness in

Table 4. Benchmark Drug data sets used for evaluation.

\begin{tabular}{llll}
\hline Index & Type & \# Drugs & \# Associations \\
\hline 1 & Enzymes & 445 & 2,926 \\
2 & lon Channels & 210 & 1,476 \\
3 & GPCRs & 223 & 635 \\
4 & Nuclear Receptors & 54 & 90 \\
\hline doi:10.1371/journal.pone.0058977.t004 & &
\end{tabular}


the "negative" samples. Let $T$ be the number of bootstraps, let $\mathcal{A}$ be the set of positives (i.e. gene-phenotype pairs that correspond to known associations), let $n_{+}$denote the number of examples in $\mathcal{A}$, and let $\mathcal{U}$ denote the set of unlabeled gene-phenotype pairs. We train a biased SVM, where we use a penalty $C_{-}$for false positives and relatively larger penalty $C_{+}$for false negatives.

The bagging algorithm that trains and combines several biased SVM classifiers used by CATAPULT is as follows:

initialize $\theta=0, s(x)=0, \forall x \in \mathcal{U}$, and $n(x)=1, \forall x \in \mathcal{U}$.

for $t=1,2, \ldots, T$ :

1..Draw a bootstrap sample $\mathcal{U}_{t} \subseteq \mathcal{U}$ of size $n_{+}$.

2..Train a linear classifier $\theta_{t}$ using the positive training examples $\mathcal{A}$ and $\mathcal{U}_{t}$ as negative examples by solving:

$$
\min _{\theta^{\prime} \in \mathbb{R}^{d}} \frac{1}{2}\left\|\theta^{\prime}\right\|^{2}+C_{-} \sum_{i \in \mathcal{U}_{t}} \xi_{i}+C_{+} \sum_{i \in \mathcal{P}} \xi_{i}
$$

subject to $\xi_{i} \geq 0, \forall i \in \mathcal{A} \cup \mathcal{U}_{t}$,

$\left\langle\Phi\left(x_{i}\right), \theta^{\prime}\right\rangle \geq 1-\xi_{i}, \forall i \in \mathcal{A}$, and

$-\left\langle\Phi\left(x_{i}\right), \theta^{\prime}\right\rangle \geq 1-\xi_{i}, \forall i \in \mathcal{U}_{t}$.

3..For any $x \in \mathcal{U} \mathcal{U}_{t}$ update:

(i) $n(x) \leftarrow n(x)+1$.

(ii) $s(x) \leftarrow s(x)+\left\langle\theta_{t}, \Phi(x)\right\rangle$.

return $s(x) \leftarrow s(x) / n(x), \forall x \in \mathcal{U}$.

We train a biased SVM given in equation (6) during each iteration using all the known positive examples in $\mathcal{A}$ and a randomly chosen set of "negatives" $\mathcal{U}_{t} \subseteq \mathcal{U}$. Positive and negative examples may not be linearly separable, and the usual approach in SVMs is to penalize each example based on how far it is from meeting its margin requirement, through the use of slack variables $\xi_{i}$. The scoring function for iteration $t$ is proportional to the distance of the point $x$ from the hyperplane and is given by the standard dot product,

\section{$\left\langle\theta_{t}, \Phi(x)\right\rangle$}

where $\theta_{t}$ is the normal to the hyperplane learned using the random bootstrap at the $t$ th iteration and $\Phi(x)$ is the feature vector corresponding to $x$. For small number of boostraps, say $T$ in the range $10-100, n(x)=T$ for most of the unlabeled examples and thus the procedure in effect scores (most of the) unlabeled examples using the average hyperplane $\frac{1}{T} \sum_{t} \theta_{t}$. We set $T=30$ in our experiments. Recall that, in our framework, an instance $x$ corresponds to a gene-phenotype pair. In contrast to the traditional SVM classifiers that classify a pair as positive or negative based on the sign of $\left\langle\theta_{t}, \Phi(x)\right\rangle$, we use the value as a score under the assumption that the further a point is on the positive side of the hyperplane, the more likely it is to be a true positive.

Parameters. In equation (6), $C_{+}$and $C_{-}$are the penalties on misclassified positives and negatives respectively. Typically, $C_{+}>C_{-}$. The weights control the relative widths of the margins on either sides of the hyperplane. As $C_{+}$increases from 0 to $\infty$, the margin on the side of the positive examples shrinks, and as $C_{+} \rightarrow \infty$, the classifier attempts to make no mistake on the positive examples. The ratio $C_{+} / C_{-}$determines the "weight" of a positive example, and we want this to be high. In our experiments, we set $C_{-}=1$ and $C_{+}=10$, which is found to be the best by crossvalidation. The cross-validation procedure for tuning parameters is given as follows:

1. Sample a fraction $(70 \%)$ of the positives from the training data points (gene-disease associations) to form the validation set $\mathcal{V}$.

2. Split the validation set into 5 folds: $\mathcal{V}_{1}, \mathcal{V}_{2}, \ldots, \mathcal{V}_{5}$.

3. $C_{-} \leftarrow 1$.

4. for $\log \left(C_{+}\right)$in $[-3,-2,-1,0,1,2,3]$ :

$$
\text { for } i=1 \text { to } 5 \text { : }
$$

(a) Fix $\mathcal{V}_{i}$ to be the test set and the remaining 4 folds, $\bigcup_{j \neq i} \mathcal{V}_{j}$, to be the training set.

(b) Train Catapult with the positive training set $\mathcal{A}=\bigcup_{j \neq i} \mathcal{V}_{j}$, and the current $\left(C_{+}, C_{-}\right)$values in equation (6). (Note that an equal number of "negative" examples are randomly sampled from the unlabeled gene-disease associations.).

(c) Obtain the recall rate $R_{i}$ of CATAPULT on the hidden test set, i.e. fraction of true positives in $\mathcal{V}_{i}$ identified in the top $k$ predictions, where $k=\left|\mathcal{V}_{i}\right|$.

Let $R_{C_{+}}=(1 / 5) \sum_{i=1}^{5} R_{i}$.

5. Return $C_{+}=\operatorname{argmax}_{C_{+}} R_{C_{+}}$and $C_{-}$.

Using the cross-validated values of $C_{+}$and $C_{-}$for a particular train-test split (Fixing $C_{-}=1, \log _{10} C_{+}$was varied in the range $-3,-2,-1,0,1,2,3$ and $\log _{10} C_{+}=1$ was typically the best), we train the model on the training data and evaluate on the test data. This is done three times corresponding to 3 random train-test splits of the full gene-disease associations data.

Features derived from hybrid walks. Before applying any supervised machine learning approach, we need to construct features for gene-phenotype pairs. The features that we use are all based on paths in the heterogeneous network. Recall that in the Katz measure, the weights for combining the contributions from walks of different lengths are fixed beforehand. We observe from equation (5) that, for a given length of walk, there are multiple ways of obtaining hybrid walks, as given by the terms in the series. For a given gene-phenotype pair, different walks of the same length, and walks of different lengths can be used as features for the pair. Thus learning a biased SVM provides an efficient way to learn the weights, and could help improve on the prediction performance over a particular choice of weights, say, $\left(\beta, \beta^{2}, \beta^{3}, \ldots\right)$ as in the Katz measure. Clearly, the dimensionality of the feature space is exponential in $k$, the length of the walk, and makes us vulnerable to the curse of dimensionality because the examples are limited. However, taking a cue from the fact that the weights of increasing walk lengths need to be heavily damped, we ignore higher order terms and thereby keep the dimensionality of the feature space small.

Relationship between the learned $\theta$ and $\beta$. The complete set of features used by CATAPULT and the corresponding weights learned are listed in Table 5. The features relate to the expression 
Table 5. Weights by CATAPULT.

\begin{tabular}{|c|c|c|c|c|}
\hline Type & Feature & Learned weights & Feature & Learned weights \\
\hline & $P_{H s} P_{H s}^{T} P_{H s}$ & 31.04 & $P_{H s} P_{H s}^{T} G^{2} P_{H s}$ & 8.97 \\
\hline \multirow[t]{3}{*}{ Human } & $P_{H s} P_{H S}^{T} G P_{H s}$ & 2.60 & $G P_{H s} P_{H S}^{T} G P_{H s}$ & 5.98 \\
\hline & $G P_{H s} P_{H s}^{T} P_{H s}$ & 1.00 & $G^{2} P_{H s} P_{H s}^{T} P_{H s}$ & 3.31 \\
\hline & $P_{A t} P_{A t}^{T} P_{H s}$ & 8.09 & $P_{A t} P_{A t}^{T} G^{2} P_{H s}$ & 0.74 \\
\hline \multirow[t]{3}{*}{ Plant } & $P_{A t} P_{A t}^{T} G P_{H s}$ & 1.06 & $G P_{A t} P_{A t}^{T} G P_{H s}$ & 2.18 \\
\hline & $G P_{A t} P_{A t}^{T} P_{H s}$ & 1.20 & $G^{2} P_{A t} P_{A t}^{T} P_{H s}$ & 0.65 \\
\hline & $P_{C e} P_{C e}^{T} P_{H s}$ & 5.75 & $P_{C e} P_{C e}^{T} G^{2} P_{H s}$ & 0.33 \\
\hline \multirow[t]{3}{*}{ Worm } & $P_{C e} P_{C e}^{T} G P_{H s}$ & 0.69 & $G P_{C e} P_{C e}^{T} G P_{H s}$ & 0.55 \\
\hline & $G P_{C e} P_{C e}^{T} P_{H s}$ & 0.62 & $G^{2} P_{C e} P_{C e}^{T} P_{H s}$ & 0.29 \\
\hline & $P_{D m} P_{D m}^{T} P_{H s}$ & 4.58 & $P_{D m} P_{D m}^{T} G^{2} P_{H s}$ & 0.90 \\
\hline \multirow[t]{3}{*}{ Fly } & $P_{D m} P_{D m}^{T} G P_{H s}$ & 0.93 & $G P_{D m} P_{D m}^{T} G P_{H s}$ & 1.36 \\
\hline & $G P_{D m} P_{D m}^{T} P_{H s}$ & 0.72 & $G^{2} P_{D m} P_{D m}^{T} P_{H s}$ & 0.55 \\
\hline & $P_{D r} P_{D r}^{T} P_{H s}$ & 8.28 & $P_{D r} P_{D r}^{T} G^{2} P_{H s}$ & 1.16 \\
\hline \multirow[t]{3}{*}{ Zebrafish } & $P_{D r} P_{D r}^{T} G P_{H s}$ & 0.77 & $G P_{D r} P_{D r}^{T} G P_{H s}$ & 2.68 \\
\hline & $G P_{D r} P_{D r}^{T} P_{H s}$ & 0.52 & $G^{2} P_{D r} P_{D r}^{T} P_{H s}$ & 0.69 \\
\hline & $P_{E c} P_{E c}^{T} P_{H s}$ & 1.67 & $P_{E c} P_{E c}^{T} G^{2} P_{H s}$ & 0.19 \\
\hline \multirow[t]{3}{*}{ E. coli } & $P_{E c} P_{E c}^{T} G P_{H s}$ & 0.30 & $G P_{E c} P_{E c}^{T} G P_{H s}$ & 0.75 \\
\hline & $G P_{E c} P_{E c}^{T} P_{H s}$ & 0.29 & $G^{2} P_{E c} P_{E c}^{T} P_{H s}$ & 0.12 \\
\hline & $P_{G g} P_{G g}^{T} P_{H s}$ & 3.76 & $P_{G g} P_{G g}^{T} G^{2} P_{H s}$ & 0.32 \\
\hline \multirow[t]{3}{*}{ Chicken } & $P_{G g} P_{G g}^{T} G P_{H s}$ & 0.30 & $G P_{G g} P_{G g}^{T} G P_{H s}$ & 1.35 \\
\hline & $G P_{G g} P_{G g}^{T} P_{H s}$ & 0.23 & $G^{2} P_{G g} P_{G g}^{T} P_{H s}$ & 1.82 \\
\hline & $P_{M m} P_{M m}^{T} P_{H s}$ & 15.03 & $P_{M m} P_{M m}^{T} G^{2} P_{H s}$ & 1.54 \\
\hline \multirow[t]{3}{*}{ Mouse } & $P_{M m} P_{M m}^{T} G P_{H s}$ & 1.35 & $G P_{M m} P_{M m}^{T} G P_{H s}$ & 2.13 \\
\hline & $G P_{M m} P_{M m}^{T} P_{H s}$ & 0.83 & $G^{2} P_{M m} P_{M m}^{T} P_{H s}$ & 0.70 \\
\hline & $P_{S c} P_{S c}^{T} P_{H s}$ & 5.55 & $P_{S c} P_{S c}^{T} G^{2} P_{H s}$ & 0.30 \\
\hline \multirow[t]{2}{*}{ Yeast } & $P_{S c} P_{S c}^{T} G P_{H s}$ & 0.61 & $G P_{S c} P_{S c}^{T} G P_{H s}$ & 0.59 \\
\hline & $G P_{S c} P_{S c}^{T} P_{H s}$ & 0.56 & $G^{2} P_{S c} P_{S c}^{T} P_{H s}$ & 0.25 \\
\hline \multirow[t]{2}{*}{ Gene network } & $G P_{H s}$ & 1.23 & $G^{3} P_{H s}$ & 0.57 \\
\hline & $G^{2} P_{H s}$ & 3.52 & $G^{4} P_{H s}$ & 0.36 \\
\hline \multirow[t]{4}{*}{ Phenotype network } & $P_{H s} Q_{H s}$ & 39.63 & $P_{H s} P_{H s}^{T} G^{2} P_{H s} Q_{H s}$ & 4.28 \\
\hline & $P_{H s} P_{H s}^{T} P_{H s} Q_{H s}$ & 21.02 & $G P_{H s} P_{H s}^{T} G P_{H s} Q_{H s}$ & 2.56 \\
\hline & $P_{H s} P_{H s}^{T} G P_{H s} Q_{H s}$ & 1.70 & $G^{2} P_{H s} P_{H s}^{T} P_{H s} Q_{H s}$ & 1.43 \\
\hline & $G P_{H s} P_{H s}^{T} P_{H s} Q_{H s}$ & 0.64 & & \\
\hline
\end{tabular}

Weights learned for different features by CATAPULT using the biased SVM with bagging procedure, using the HumanNet gene network. Two important observations are: (1) Features corresponding to longer path lengths receive relatively much smaller weights (note that path length can be deduced from the number of terms in the feature, for example, $P_{H s} P_{H s}^{T} P_{H s}$ has path length 3, while $P_{H s} P_{H s}^{T} G P_{H s}$ has path length 4). (2) Features corresponding to different species receive different weights, in particular, features derived from mouse phenotypes get the highest weights, which makes sense given the relative evolutionary proximity between humans and mice. The relative weights of different information sources are not straightforward to interpret. However, we can see that some higher order features are informative.

doi:10.1371/journal.pone.0058977.t005

for the Katz method given in (5). In particular, the term involving $P P^{T} \quad$ can be written as $P P^{T}=\sum_{i \in \mathcal{S}} P_{i} P_{i}^{T}$, where $\mathcal{S}=\{H s, A t, C e, D m, D r, E c, G g, M m, S c\}$. The Katz method weighs terms involving paths of length $l$ by $\beta^{l}$, and does not distinguish between species or between sources. As an example of distinguishing by source, consider the terms corresponding to $l=2$ in the expression (5), i.e. $G P_{H s}$ and $P_{H s} Q_{H s}$. The Katz method weighs both the types of paths by $\beta^{2}$ whereas the CATAPULT method learns two different weights $\theta_{i}=1.23$ and $\theta_{j}=39.63$ corresponding to the two features, as observed from Table 5. For distinguishing by species, consider the term $P P^{T} P_{H_{s}}$ corresponding to $l=3$ in (5). In this case, Katz method uses $\beta^{3}$, whereas CATApult learns a set of 9 different weights corresponding to different species, i.e. $P P^{T} P_{H s}=\sum_{i \in \mathcal{S}} P_{i} P_{i}^{T} P_{H s}$. We observe from Table 5 that different species contribute differently towards the final prediction. Furthermore, we also observe from our experiments using specieswise features not only lends interpretability but also improves the accuracy of the predictions, as compared to combining features corresponding to same walk lengths (Note that all CATAPULT results 
shown in the paper use the features listed in Table 5, and results for combining features are not shown).

Random Walks with Restart on Heterogeneous network. Random Walks with Restart on Heterogeneous network (RWRH) is an algorithm for predicting gene-disease associations proposed by Li and Patra [8]. RWRH performs a random walk on a heterogeneous network of gene interactions (HPRD) and human diseases (we used OMIM phenotypes and the drug data described above). The method constructs a heterogeneous network using $G^{H P R D}, P_{H s}$ and $Q_{H s}$ networks and runs a personalized PageRank computation, a popular choice for ranking documents and web pages, on the network. The random walk is started from a set of seed nodes, which for a phenotype $p$ is the set of genes known to be associated with $p$, and gene nodes are ranked by the probability that a random walker is at a given gene, under the steady state distribution for the random walk. In particular, RWRH [8] considers the following heterogeneous network:

$$
\tilde{C}=\left[\begin{array}{cc}
\tilde{G} & \lambda P_{H s} \\
\lambda P_{H s}^{\mathrm{T}} & \tilde{Q}_{H s}
\end{array}\right]
$$

where $\tilde{G}$ is the gene-gene interactions matrix, $\tilde{Q}_{H s}$ is the phenotype-phenotype similarity matrix, and $\lambda$ is the probability that the random walker jumps from a gene node to a phenotype node (or vice versa). The matrices $\tilde{G}, \tilde{Q}_{H s}, P_{H s}$ and $P_{H s}^{T}$ are normalized by row-degree. The rows of the matrices $\tilde{G}$ and $\tilde{Q}_{H s}$ scaled by a factor of $(1-\lambda)$, as appropriate, so that $\tilde{C}$ is a rowstochastic matrix, i.e. $\sum_{j} \tilde{C}_{i j}=1, \forall i$. In [8], $P_{H s}$ is the gene-disease association matrix corresponding to OMIM phenotypes, $\tilde{Q}_{H s}$ is the corresponding similarity matrix, and $\tilde{G}$ is derived from $G^{H P R D}$. Genes are ranked for a given disease $p$ using the steady state vector $\mathbf{s}^{*}$, the solution to the equation:

$$
\mathbf{s}=(1-\gamma) \tilde{C}^{T} \mathbf{s}+\gamma p_{0}
$$

where $p_{0}$ is the restart vector (indicator vector of the set of seed nodes known to be associated with $p$ ) and $\gamma$ is the restart probability. In our experiments, we use OMIM phenotypes matrix $P_{H s}$ as well as the gene-drug interaction matrix $D$, and two types of gene-gene matrices to derive $\tilde{G}$. Recall that in the latter case, we do not have similarity information for drugs, and therefore we set drug-drug similarity matrix to 0 . It is also straightforward to incorporate phenotype data from multiple species in the method, by replacing $P_{H s}$ with $P=\left[\begin{array}{ll}P_{H s} & P_{S}\end{array}\right]$, analogous to our Katz method.

PRINCE. The PRINCE method, proposed by Vanunu et al. [7], is another graph-based method that can be thought of as a special case of RWRH. Here, the random walk is only over the gene interaction network instead of the heterogeneous network. The phenotype similarities are incorporated in the restart vector. The vector of scores computed by PRINCE for a given phenotype $p$ can be expressed as

$$
\mathbf{s}_{P R I N C E}=(I-\gamma G)^{-1} \tilde{p}
$$

where $\tilde{p}$ is the smoothed phenotype, i.e. $\tilde{p}_{i}=\left(Q_{H s}\right)_{q p}$ where $q$ is the phenotype most similar to $p$ such that gene $i$ is known to be associated with disease $q$ and $\gamma$ is the restart probability. Note that, similarly, the scores computed by RWRH can be written succinctly as

$$
\mathbf{s}_{R W R H}=(I-\gamma \tilde{C})^{-1} p
$$

where $\tilde{C}$ is defined in equation (7). We show the relationship between the Katz method and other random-walk based methods (PRINCE and RWRH) in the Text S1. The absence of similarity information for other (non-human) species phenotypes and drugs renders direct extension of PRINCE to multiple species data inconsequential. We must emphasize here that PRINCE does not allow walks through the gene-phenotype interaction network or the phenotype-phenotype interaction network. As a result, availability of other species data becomes irrelevant when predicting genes for a given disease (or other drug data in case of predicting for a given drug).

ProDiGe. The ProDiGe method, proposed by Mordelet and Vert [15], makes use of positive-unlabeled learning and a multiple kernel learning framework to integrate information from multiple types of gene interaction data and phenotype similarities. Kernels are defined over pairs of genes and pairs of phenotypes, and the kernel value for a pair of gene-phenotype pairs is derived using the individual gene and phenotype kernels. In particular, let $K_{\text {gene }}\left(g, g^{\prime}\right)$ denote the kernel for genes, and $K_{\text {phenotype }}\left(p, p^{\prime}\right)$ denote that for phenotypes. Then, the kernel for the pairs $\left((g, p),\left(g^{\prime}, p^{\prime}\right)\right)$ is simply,

$$
K_{\text {pair }}\left((g, p),\left(g^{\prime}, p^{\prime}\right)\right)=K_{\text {gene }}\left(g, g^{\prime}\right) \times K_{\text {phenotype }}\left(p, p^{\prime}\right)
$$

The gene-phenotype pairs are then classified using a support vector machine using the constructed kernel. Note that ProDiGe [15] does not use any other species phenotype information, but only the OMIM phenotypes. In our experiments on OMIM phenotypes, we used the kernels $K_{\text {gene }}$ and $K_{\text {phenotype }}$ provided by Mordelet [15] downloaded from:

http://cbio.ensmp.fr/jvert/svn/prodige/html/prodige-0.3.tar. gz. For the drug data, we used a simple Dirac kernel (since kernel matrices need to be positive definite) for $K_{d r u g}\left(d, d^{\prime}\right)$, i.e.

$$
K_{d r u g}\left(d, d^{\prime}\right)=\left\{\begin{array}{cc}
1 & \text { if } d=d^{\prime} \\
0 & \text { otherwise }
\end{array}\right.
$$

\section{Implementation}

We implemented all the methods in Matlab. Our implementation of Catapult can be downloaded from the Catapult home page: http://marcottelab.org/index.php/Catapult. A web interface for querying recommendations for a given phenotype is also accessible from the page. Obtaining features for all genephenotype pairs takes about 20 minutes. Training Catapult is much faster, and takes a few seconds per iteration of the algorithm on our cluster machines (2.8 $\mathrm{GHz}$ processor, 32GB RAM). The sourcecodes for Li and Patra's RWRH method and ProDiGe were obtained from the respective project home pages http://www3. ntu.edu.sg/home/aspatra/research/Yongjin_BI2010.zip and http://cbio.ensmp.fr/jvert/svn/prodige/html/prodige-0.3.tar.gz. For PRINCE, we use MATLAB code kindly provided by Oded Magger. 


\section{Supporting Information}

Text S1 A detailed derivation of the relationship between Katz on the heterogeneous network, PRINGE, and RWRH.

(PDF)

\section{Acknowledgments}

The authors want to thank Jon Laurent and Kris McGary for some of the data used, and $\mathrm{Li}$ and Patra for making their code available. Most of

\section{References}

1. Goh K, Cusick M, Valle D, Childs B, Vidal M, et al. (2007) The human disease network. Proceedings of the National Academy of Sciences 104: 8685.

2. Tian W, Zhang LV, Taan M, Gibbons FD, King OD, et al. (2008) Combining guilt-by-association and guilt-by-profiling to predict Saccharomyces cerevisiae gene function. Genome Biology 9 Suppl 1: S7.

3. Ulitsky I, Shamir R (2007) Identification of functional modules using network topology and highthroughput data. BMC systems biology 1: 8

4. Human Protein Reaction Database, HPRD. Available: http://www.hprd.org. Accessed: 2012 Aug.

5. Wu X, Jiang R, Zhang MQ, Li S (2008) Network-based global inference of human disease genes. Mol Syst Biol 4: 189.

6. Köhler S, Bauer S, Horn D, Robinson PN (2008) Walking the Interactome for Prioritization of Candidate Disease Genes. The American Journal of Human Genetics: 949-958.

7. Vanunu O, Magger O, Ruppin E, Shlomi T, Sharan R (2010) Associating genes and protein complexes with disease via network propagation. PLoS computational biology 6: e1000641.

8. Li Y, Patra JC (2010) Genome-wide inferring gene-phenotype relationship by walking on the heterogeneous network. Bioinformatics/computer Applications in The Biosciences 26: 1219-1224.

9. Lee I, Date SV, Adai AT, Marcotte EM (2004) A probabilistic functional network of yeast genes. Science 306: 1555-1558.

10. McGary KL, Lee I, Marcotte EM (2007) Broad network-based predictability of Saccharomyces cerevisiae gene loss-of-function phenotypes. Genome biology 8: R258.

11. Mostafavi S, Ray D, Warde-Farley D, Grouios C, Morris Q (2008) GeneMANIA: a real-time multiple association network integration algorithm for predicting gene function. Genome biology 9 Suppl 1: S4

12. Huttenhower C, Haley EM, Hibbs MA, Dumeaux V, Barrett DR, et al. (2009) Exploring the human genome with functional maps. Genome Research 19: 1093-1106.

13. Linghu B, Snitkin ES, Hu Z, Xia Y, Delisi G (2009) Genome-wide prioritization of disease genes and identification of disease-disease associations from an integrated human functional linkage network. Genome Biology 10: R91.

14. Lee I, Blom UM, Wang PI, Shim JE, Marcotte EM (2011) Prioritizing candidate disease genes by network-based boosting of genome-wide association data. Genome research 21: 1109-21.

15. Mordelet F, Vert JP (2011) Prodige: Prioritization of disease genes with multitask machine learning from positive and unlabeled examples. BMC Bioinformatics 12.

16. McGary KL, Park TJ, Woods JO, Cha HJ, Wallingford JB, et al. (2010) Systematic discovery of nonobvious human disease models through orthologous phenotypes. Proceedings of the National Academy of Sciences of the United States of America 107: 6544-9.

17. Katz L (1953) A new status index derived from sociometric analysis. Psychometrika 18: 39-43.

18. Gillis J, Pavlidis P (2011) The Impact of Multifunctional Genes on "Guilt by Association" Analysis. Plos One 6.

19. Cheng F, Liu C, Jiang J, Lu W, Li W, et al. (2012) Prediction of Drug-Target Interactions and Drug Repositioning via Network-Based Inference. PLoS Comput Biol 8: e1002503+.

20. Liben-Nowell D, Kleinberg J (2007) The link-prediction problem for social networks. Journal of the American Society for Information Science and Technology 58: 1019-1031.

21. Estrada E, Higham DJ (2010) Network properties revealed through matrix functions. SIAM Rev 52: 696-714.

22. Page L, Brin S, Motwani R, Winograd T (1999) The pagerank citation ranking: Bringing order to the web.

23. Lu Z, Savas B, Tang W, Dhillon I (2010) Supervised link prediction using multiple sources. In: Data Mining (ICDM), 2010 IEEE 10th International Conference on. IEEE, pp. 923-928.

24. Liu B, Dai Y, Li X, Lee WS, Yu PS (2003) Building text classifiers using positive and unlabeled examples. In: Intl. Conf. on Data Mining. pp. 179-188.

25. Mordelet F, Vert JP (2010) A bagging SVM to learn from positive and unlabeled examples. Technical Report hal-00523336, version 1, HAL.
Ambuj Tewari's contribution to this work happened while he was a postdoctoral fellow at the University of Texas at Austin.

\section{Author Contributions}

Conceived and designed the experiments: UMS-B NN AT ISD EMM. Performed the experiments: UMS-B NN. Analyzed the data: UMS-B NN. Contributed reagents/materials/analysis tools: JOW. Wrote the paper: UMS-B NN AT ISD EMM.

26. Lee WS, Liu B (2003) Learning with positive and unlabeled examples using weighted logistic regression. In: Proceedings of the Twentieth International Conference on Machine Learning (ICML). pp. 448-455.

27. Online Mendelian Inheritance in Man, OMIM (2011) Available: http://omim. org/. Accessed: 2011 Aug.

28. Van Driel M, Bruggeman J, Vriend G, Brunner H, Leunissen J (2006) A textmining analysis of the human phenome. European journal of human genetics 14: 535-542.

29. Mostafavi S, Morris Q (2010) Fast integration of heterogeneous data sources for predicting gene function with limited annotation. Bioinformatics (Oxford, England) 26: 1759-65.

30. Cannata D, Fierz Y, Vijayakumar A, LeRoith D (2010) Type 2 diabetes and cancer: what is the connection? The Mount Sinai journal of medicine New York 77: 197-213.

31. Hoe HS, Rebeck GW (2008) Functional interaction of APP with the apoE receptor family. Journal of Neurochemistry 106: 2263-71.

32. Van Helmond ZK, Miners JS, Bednall E, Chalmers KA, Zhang Y, et al. (2007) Caveolin-1 and -2 and their relationship to cerebral amyloid angiopathy in Alzheimer's disease. Neuropathology and Applied Neurobiology 33: 317-327.

33. Jong YJI, Dalemar LR, Seehra K, Baenziger NL (2002) Bradykinin receptor modulation in cellular models of aging and Alzheimer's disease. International Immunopharmacology 2: 1833-1840.

34. Le Beau MM, Larson RA, Bitter MA, Vardiman JW, Golomb HM, et al. (1983) Association of an inversion of chromosome 16 with abnormal marrow eosinophils in acute myelomonocytic leukemia. A unique cytogenetic-clinicopathological association. New England Journal of Medicine 309: 630-636.

35. Molnar S, Mihanović M, Grah M, Kezić S, Filaković P, et al. (2010) Comparative study on gene tags of the neurotransmission system in schizophrenic and suicidal subjects. Collegium Antropologicum 34: 1427-1432.

36. Peri S, Navarro JD, Amanchy R, Kristiansen TZ, Jonnalagadda CK, et al. (2003) Development of human protein reference database as an initial platform for approaching systems biology in humans. Genome research 13: 2363-71.

37. Karni S, Soreq H, Sharan R (2009) A network-based method for predicting disease-causing genes. Journal of Computational Biology 16: 181-189.

38. Swarbreck D, Wilks C, Lamesch P, et al. (2008) The Arabidopsis Information Resource (TAIR): gene structure and function annotation. Nucleic acids research 36: D1009-14.

39. Chen N, Harris TW, Antoshechkin I, et al. (2005) WormBase: a comprehensive data resource for Caenorhabditis biology and genomics. Nucleic acids research 33: D383-9.

40. Green RA, Kao HL, Audhya A, Arur S, Mayers JR, et al. (2011) A HighResolution C. elegans Essential Gene Network Based on Phenotypic Profiling of a Complex Tissue. Cell 145: 470-482.

41. Tweedie S, Ashburner M, Falls K, Leyland P, McQuilton P, et al. (2009) FlyBase: enhancing Drosophila Gene Ontology annotations. Nucleic acids research 37: D555-9.

42. Eppig JT, Blake JA, Bult CJ, Kadin JA, Richardson JE (2007) The mouse genome database (MGD): new features facilitating a model system. Nucleic acids research 35: D630-7.

43. Dwight SS, Harris MA, Dolinski K, Ball GA, Binkley G, et al. (2002) Saccharomyces Genome Database (SGD) provides secondary gene annotation using the Gene Ontology (GO). Nucleic acids research 30: 69-72.

44. Saito TL, Ohtani M, Sawai H, Sano F, Saka A, et al. (2004) SCMD: Saccharomyces cerevisiae Morphological Database. Nucleic acids research 32: D319-22.

45. Hillenmeyer ME, Fung E, Wildenhain J, Pierce SE, Hoon S, et al. (2008) The chemical genomic portrait of yeast: uncovering a phenotype for all genes. Science (New York, NY) 320: 362-5.

46. Nichols RJ, Sen S, Choo YJ, Beltrao P, Zietek M, et al. (2011) Phenotypic landscape of a bacterial cell. Cell 144: 143-56.

47. Sprague J, Bayraktaroglu L, Clements D, Conlin T, Fashena D, et al. (2006) The Zebrafish Information Network: the zebrafish model organism database. Nucleic acids research 34: D581-5.

48. Bell GW, Yatskievych TA, Antin PB (2004) GEISHA, a whole-mount in situ hybridization gene expression screen in chicken embryos. Developmental dynamics: an official publication of the American Association of Anatomists 229: $677-87$. 
49. Remm M, Storm CE, Sonnhammer EL (2001) Automatic clustering of orthologs and in-paralogs from pairwise species comparisons. Journal of molecular biology 314: $1041-52$.
50. Yamanishi Y, Araki M, Gutteridge A, Honda W, Kanehisa M (2008) Prediction of drug-target interaction networks from the integration of chemical and genomic spaces. In: ISMB. pp. 232-240.

51. Molecular Modeling and Design, LMMD. Available: http://www.lmmd.org/ database/dti. Accessed 2012 Aug. 